\title{
Tanulmány
}

András Kertész \& Csaba Földes

\section{Von Humboldt zur IT. Germanistische Forschung und Lehre in Ungarn: \\ Tendenzen und Perspektiven im Referenzsystem des europäischen Hochschulraums}

\begin{abstract}
The paper discusses the present state of the art of German studies in Hungary in the context of the main tendencies in the European higher education area. As a point of departure, we give a brief overview of substantial developments in the European higher education area. Then, a brief analysis follows which reveals some main features of the current situation of the humanities in Hungary resulting from the European developments. As a third step, our train of thought is further narrowed down by relating the findings of this analysis to the current tendencies in and perspectives of German studies in the Hungarian higher education system. Fourth, the overall picture that emerges in this way is supplemented by two empirical surveys. The final section sketches some of the strategies that may contribute to overcoming the difficulties with which German studies in Hungary have been confronted.

Keywords: German studies, European higher education area, multingualism, humanities, Hungarian higher education system
\end{abstract}

\section{$1 \quad$ Aufgabenstellung}

Die aktuelle Situation des akademischen Faches Germanistik gestaltet sich weltweit recht unterschiedlich: Es sind markante Auf- und Abschwünge des Deutschen als nationale und internationale Verkehrs-, Kultur-, Wissenschafts- und Fachsprache sowie als Fremd- und Zweitsprache zu konstatieren. Während das Fach in manchen Ländern eine drastische Reduzierung von Lehrstühlen erlebt (z.B. in Russland), können in anderen Staaten die Germanistik und Deutsch als Fremdsprache regelrecht boomen (z.B. in China): Das Deutschstudium bzw. der Erwerb der deutschen Sprache ist hoch angesehen und dient als Eintrittskarte zum beruflichen Aufstieg. Allerdings gibt es auch Regionen, in denen damit die Befürchtung verbunden ist, dass durch das Erlernen des Deutschen die gut qualifizierte und mobile Schicht 


\author{
András Kertész \& Csaba Földes: \\ Von Humboldt zur IT. Germanistische Forschung und Lehre in Ungarn: \\ Tendenzen und Perspektiven im Referenzsystem des europäischen Hochschulraums \\ Argumentum 15 (2019), 799-824 \\ Debreceni Egyetemi Kiadó \\ DOI: $10.34103 / A R G U M E N T U M / 2019 / 6$
}

der Bevölkerung nach Deutschland, Österreich oder in die Schweiz abwandert (z.B. in einigen Balkan-Ländern). ${ }^{1}$

Vor diesem globalen Hintergrund setzt sich der vorliegende Beitrag zum Ziel, zumindest vorläufige Antworten auf folgende spezielle Fragen zu erarbeiten:

(1) Was sind die Haupttendenzen der gegenwärtigen ungarischen Germanistik im Kontext des europäischen Hochschulraums?

(2) Welche Schlussfolgerungen ergeben sich daraus im Hinblick auf Maßnahmen, die die ungarische Germanistik - in ihrem eigenen Interesse - treffen sollte?

Zur Beantwortung dieser beiden Fragen werden wir unsere Überlegungen schrittweise spezifizieren. In einem ersten Schritt soll ein relativ abstrakter und allgemeiner Überblick zu den zentralen Linien der Veränderungen im europäischen Hochschulsystem als Hintergrund herausgearbeitet werden. Auf dieser Basis skizzieren wir dann die Auswirkung dieser Tendenzen auf die aktuelle Situation der Geisteswissenschaften in Ungarn. In einem dritten Schritt sollen unsere Erwägungen zu den Geisteswissenschaften auf Tendenzen und Perspektiven der ungarischen Germanistik hin spezifiziert werden. In einem vierten Schritt möchten wir das Bild, das sich dabei entfaltet, um die Auswertung zweier empirischer Umfragen sowohl ergänzen als auch differenzieren. Schließlich sollen entsprechende Schlussfolgerungen gezogen werden.

\title{
2 Tendenzen im europäischen Hochschulraum
}

Das Humboldtsche Modell europäischer Universitäten, das sich im 19. Jahrhundert herausgebildet hat und bis zum heutigen Tag mehr oder weniger den institutionellen Rahmen für Forschung und Lehre an Universitäten darstellt, ist u.a. durch eine Autonomie von Professoren bzw. ihrer Lehrstühle gekennzeichnet. Wissenschaftler werden vor allem dadurch motiviert, dass sie durch ihre Forschungstätigkeit hohe Reputation erlangen können. Im Wertesystem des Humboldtschen Konzepts von Forschung und Lehre steht die Anerkennung seitens der Fachkollegen an erster Stelle; was für Wissenschaftler wirklich zählt, ist folglich - etwas vereinfacht dargestellt - in erster Linie nicht Gewinnstreben, sondern wissenschaftlicher Ruhm, die Wertschätzung der intellektuellen Leistung sowie die Verbreitung der eigenen Ideen unter gleichgesinnten Fachkollegen und Nachwuchswissenschaftlern auf nationaler sowie internationaler Ebene (Polónyi 2005 und Pléh 2006). ${ }^{2}$ Forschungen finden - ohne instituts-, fakultäts- oder universitätsübergreifende Strategie - in der Regel aufgrund des individuellen Interesses und der persönlichen Initiative von Wissenschaftlern statt. Das höchste Ansehen genießen die Ergebnisse in der Grundlagenforschung, die in renommierten internationalen Zeitschriften (und/oder Monografien) veröffentlicht und anschließend oft zitiert wer-

Siehe zum Thema ausführlicher den Aufsatz von Földes (2016) und den von Goltschnigg (2017) herausgegebenen Sammelband.

2 Da wir in diesem Beitrag die Situation germanistischer Forschung im Lichte der ungarischen Hochschulpolitik thematisieren, zitieren wir vorwiegend ungarische Fachliteratur. Unsere Überlegungen zur Krise des Humboldtschen Modells europäischer Universitäten sowie zur Situation der Geisteswissenschaften in Ungarn beruhen teilweise auf Kertész (2008). 


\author{
András Kertész \& Csaba Földes: \\ Von Humboldt zur IT. Germanistische Forschung und Lehre in Ungarn: \\ Tendenzen und Perspektiven im Referenzsystem des europäischen Hochschulraums \\ Argumentum 15 (2019), 799-824 \\ Debreceni Egyetemi Kiadó \\ DOI: 10.34103/ARGUMENTUM/2019/6
}

den, wobei praktische Umsetzung und Gewinnorientierung keine - oder kaum eine - Rolle spielen.

Diese Humboldtsche Vision von Forschung und Lehre geriet in der zweiten Hälfte des 20. Jahrhunderts in eine Krise, die durch zeitgleich entstandene wirtschaftliche Krisen teilweise ausgelöst und teilweise weiter vertieft wurde (vgl. Polónyi 2005). Letztere zeigten sich u. a. darin, dass der Staat auf seine Dominanz bei der Verteilung finanzieller Ressourcen in den Bereichen Wissenschaft, Kultur und Ausbildung immer mehr verzichtete, wodurch sich die Mechanismen der Finanzierung mancher Institutionen radikal veränderten. Eine erste das Hochschulsystem betreffende wichtige Konsequenz dieser Veränderungen ist, dass die - auf diese Weise zurückgefahrene - staatliche Finanzierung der Universitäten zunehmend auf messbaren Leistungsparametern beruht. Die zweite Konsequenz, welche die tief greifende Neustrukturierung des finanziellen Umfelds von Wissenschaft kennzeichnet, besteht im Folgenden: Da der Staat die Finanzierung teilweise außeruniversitären Quellen überlässt, müssen sich die Institutionen selbst bemühen, den ihnen entzogenen Teil der einstigen staatlichen Finanzierung durch Drittmittel, durch Kooperationen mit der Wirtschaft oder durch die Erfüllung von außeruniversitären Forschungs- und Entwicklungsaufträgen zu kompensieren. Dadurch werden die Gewinnorientierung sowie der Zwang zur selbstständigen wirtschaftlichen Tätigkeit zu einem neuen Schwerpunkt von Hochschuleinrichtungen.

In dieser neuen Konstellation unterliegt auch das Motivationssystem einem Wandel. Während im Humboldtschen Universitätsmodell der primäre Motivationsfaktor, wie bereits erwähnt, die in der Grundlagenforschung erzielte hohe wissenschaftliche Reputation war, wird jetzt die Erwartung des Staates an die Universitäten, wirtschaftlich zu sein, durch das jeweilige Zusammenspiel folgender Faktoren bestimmt: (a) die nach wie vor existierende, aber nicht mehr vorherrschende traditionelle akademische Reputation; (b) die bürokratische Koordination (staatliche Regelung, interuniversitäre Machtverhältnisse) und (c) der Markt.

Die Spannung zwischen diesen drei Faktoren mündet allmählich in die Verdrängung des Humboldtschen Konzepts und generiert folgende drei neue Typen von Universitäten (Polónyi 2005):

- Typ 1: Wirtschaftlichen Zwängen wird nur im minimal erforderlichen Maße nachgegeben, vielmehr werden die grundlegenden Werte traditioneller Forschungsuniversitäten bewahrt: Diese bleiben - in einer differenzierten und abgeschwächten Form - dominant.

- Typ 2: Es bildet sich durch die Dominanz der bürokratischen Koordination ein neuer Typ von Dienstleistungsuniversitäten heraus, die wirtschaftliche Tätigkeit ausüben und Dienstleistungen anbieten. Die Universitätsleitung geht von führenden Akademikern zu einem professionellen Management über, das mit den Interessen wirtschaftlicher Unternehmen eng verflochten ist.

- Typ 3: Es entwickeln sich unternehmerische Universitäten. Sie sind in der Lage, Forschung zu organisieren, die unmittelbaren wirtschaftlichen, vor allem industriellen, Interessen dient. Im Rahmen vielfältiger Kooperationen mit der Wirtschaft werden sie den Anforderungen des Marktes gerecht und fördern die Ausprägung von Forschung, die zur Herstellung neuer Produkte führt, diese nach den Gesetzen des Marktes vertreibt und somit Gewinn erwirtschaftet. Universitäten dieses Typs sind weitgehend unabhängig von staatlicher Unterstützung und weisen strukturelle und funktionale Merkmale eines Großunternehmens auf. 


\author{
András Kertész \& Csaba Földes: \\ Von Humboldt zur IT. Germanistische Forschung und Lehre in Ungarn: \\ Tendenzen und Perspektiven im Referenzsystem des europäischen Hochschulraums \\ Argumentum 15 (2019), 799-824 \\ Debreceni Egyetemi Kiadó \\ DOI: 10.34103/ARGUMENTUM/2019/6
}

Dass auch in Ungarn die Umgestaltung des traditionellen Humboldtschen Systems in diesen drei Richtungen begonnen hat, konnten alle am Hochschulbetrieb Beteiligten in den Jahren 2004 bis 2006 im Zusammenhang mit den sehr heftigen Diskussionen um den damaligen Entwurf des neuen Hochschulgesetzes erleben, dessen Zielsetzung es war, die Herausbildung von unternehmerischen Universitäten zu beschleunigen. Dass dieser Versuch in seiner ursprünglichen radikalen Form scheiterte, ist ein Zeichen dafür, dass in Ungarn die Bedingungen für Dienstleistungs-, geschweige denn für unternehmerische Universitäten nicht gegeben sind. Dieses Ausgangsproblem schien sich ein Jahrzehnt später zu wiederholen, als das auch heute gültige Hochschulgesetz in Kraft trat und das Primat von Innovation und wirtschaftsnahen Studiengängen sowie die unmittelbare ökonomische Aktivität der Universitäten in den Vordergrund stellte. Folglich ist nunmehr eine typische Krisensituation entstanden: Infolge globaler gesellschaftlich-wirtschaftlicher Entwicklungen - die den gesamten europäischen Hochschulraum betreffen - sind auf der einen Seite Universitäten traditionellen Humboldtschen Typs nicht mehr in der Lage, ihre definitorischen Aufgaben gemäß Forschung und Lehre zu garantieren. Dabei sind aber auch die Voraussetzungen für die Entwicklung dienstleistender Universitäten bzw. unternehmerischer Universitäten nicht vorhanden. In dieser Gemengelage passen sich manche Universitäten, Fakultäten, Disziplinen, Forschungsverbünde oder sogar einzelne Forscherpersönlichkeiten bzw. Forschergruppen den ständig wechselnden äußeren Erwartungen, den gesellschaftlichen Herausforderungen sowie ihren eigenen finanziellen Interessen stärker an, während andere langsamer reagieren und dadurch Gefahr laufen, unterzugehen. Auf eine einfache Formel gebracht: Diejenigen, die die neue Situation erkennen, haben gute Chancen zu überleben, diejenigen aber, die an der durch das neue Umfeld nicht mehr bevorzugten akademischen Mentalität festhalten, gefährden ihre eigene Existenz sowie die Zukunft des eigenen Fachgebietes. Bemerkenswert ist dabei, dass der Ausgang dieses Kampfes nicht unmittelbar mit intellektueller Leistung, mit kulturellen Werten und der wissenschaftlichen Relevanz der Forschungsergebnisse nach Humboldtschem Maßstab zusammenhängt. Es kann durchaus sein, dass das Wertvolle, aber den neuen Anforderungen nicht effektiv genug Angepasste verdrängt wird, während das weniger Wertvolle, aber mit den breiteren gesellschaftlichen Vorgängen effektiv Verbundene sich weiterentwickelt. Denkbar ist auch, dass u. U. das Gegenteil passiert: Nämlich, dass das in welchem Sinne auch immer Anspruchsvolle durch gutes Wissenschaftsmanagement einen Weg findet, zu überleben.

\title{
3 Hochschulpolitische Tendenzen in Ungarn
}

Im Einklang mit der generellen europäischen Tendenz wird die staatliche Unterstützung geisteswissenschaftlicher Forschung und Lehre in Ungarn vor allem mit der Begründung radikal gekürzt, diese sei nicht unmittelbar wirtschaftlich verwertbar und Gewinn bringend. Dies geht mit dem Ansehensverlust der Geisteswissenschaften einher. Bezüglich der sehr komplexen Tendenz zur Förderung profitorientierter und zur Verdrängung nicht-profitorientierter Forschung, die von globalen wirtschaftlichen Vorgängen, von vielschichtigen hochschul-, wissenschafts- und kulturpolitischen Maßnahmen sowie sozialpolitischen Entscheidungen nicht $\mathrm{zu}$ trennen ist, möchten wir auf eine sehr vereinfachte und zugespitzte Weise folgende - aus dem gültigen Hochschulgesetz, aus Entscheidungen der Regierung und der zuständigen 


\author{
András Kertész \& Csaba Földes: \\ Von Humboldt zur IT. Germanistische Forschung und Lehre in Ungarn: \\ Tendenzen und Perspektiven im Referenzsystem des europäischen Hochschulraums \\ Argumentum 15 (2019), 799-824 \\ Debreceni Egyetemi Kiadó \\ DOI: 10.34103/ARGUMENTUM/2019/6
}

Ministerien sowie aus den Medien bekannte - Richtlinien der ungarischen Wissenschaftspolitik hervorheben:

(a) Der Staat ist bestrebt, die Finanzierung der Forschung zunehmend dem Markt zu überlassen. Der Schlüsselbegriff der neuen wissenschaftspolitischen Terminologie ist die Innovation. Darunter wird in diesem Zusammenhang die Entwicklung marktfähiger Produkte in enger Zusammenarbeit von Universitäten und Firmen verstanden (Polónyi 2005 und Pritz et al. 2005). Die Forschungen, die diesem Ideal der Innovation entsprechen, werden mithilfe unterschiedlicher Mittel, aber vor allem durch Projektfinanzierungen unterstützt, während diejenigen, die keinen unmittelbaren ökonomischen Nutzen versprechen, eine deutliche Benachteiligung erfahren. Somit sind Forschung und Lehre an geisteswissenschaftlichen Fakultäten in Ungarn den jeweiligen aktuell-politischen Gesichtspunkten ausgeliefert, wobei das, was wissenschaftliche Erkenntnis voranbringt, in den meisten Fällen nicht mit tagespolitischen und wirtschaftlichen Interessen im Einklang steht bzw. von den Entscheidungsträgern oft nicht einmal verstanden wird. Dadurch kann ein Gegensatz zwischen dem wissenschaftlich Fortschrittlichen und dem gesellschaftlich Nützlichen entstehen, der der wissenschaftstheoretischen Binsenweisheit widerspricht, nach der es ohne Grundlagenforschung keine angewandte Forschung geben kann. Im Besonderen stuft diese Sicht die Geisteswissenschaften als „nutzlos“ ein und schränkt ihre Möglichkeiten sowohl im Hinblick auf die staatliche Förderung als auch auf die Projektfinanzierung ein.

(b) Dadurch kristallisiert sich eine neue disziplinäre Trennlinie heraus. Während in den vergangenen Jahrzehnten Wissenschaft $u$. a. durch den Unterschied zwischen den Geistesund den Naturwissenschaften und neuerdings durch die Dreiteilung Natur-, Geistes- und Gesellschaftswissenschaften geprägt war, scheinen in diesem Kontext die Geisteswissenschaften zusammen mit der naturwissenschaftlichen Grundlagenforschung auf der einen und profitorientierte angewandte Forschung im Sinne von „Innovation“, die sowohl die angewandten Naturwissenschaften als auch die angewandten Gesellschaftswissenschaften umfasst, auf der anderen Seite zu stehen. ${ }^{3}$

(c) Trotz der ausgeprägten Präferenz der Wissenschaftspolitik für angewandte Wissenschaften und deren Kooperation mit Wirtschaftsunternehmen ist die Situation der Grundlagenforschung in den Naturwissenschaften etwas positiver einzuschätzen als die der Geisteswissenschaften. Zwar wird die staatliche Finanzierung der Ersteren ebenfalls radikal gesenkt, aber es gibt doch noch etwas mehr Möglichkeiten zur Rettung der Forschungen als in den Geisteswissenschaften - vor allem Drittmittel, um welche die Forschergruppen ansuchen können. In den Geisteswissenschaften ist dagegen selbst die Chance, an Drittmittel heranzukommen, sehr eingeschränkt. Zum einen gibt es sowohl auf nationaler als auch auf internationaler Ebene wesentlich weniger Projektausschreibungen; zum anderen sind die zur Verfügung stehenden Beträge selbst auf Gebieten, auf denen es überhaupt Antragsmöglichkeiten gibt, von vornherein wesentlich niedriger als in den Naturwissenschaften.

(d) In Ungarn ist der für Forschung und Hochschulwesen vorgesehene Teil des Bruttoinlandsprodukts im europäischen Vergleich ganz besonders niedrig, wodurch Forschung und Lehre an Universitäten noch stärker gefährdet sind als in vielen anderen EU-Mitgliedstaaten. Was die Unterstützung der geisteswissenschaftlichen Forschung angeht, ergibt sich folgendes

3 Siehe z. B. Enyedi (2001 und 2004) über die Konsequenzen der Tatsache, dass in den Geisteswissenschaften die Unterscheidung zwischen Grundlagenforschung und angewandter Forschung nicht nach denselben Kriterien vorgenommen werden kann wie in den Naturwissenschaften. 


\author{
András Kertész \& Csaba Földes: \\ Von Humboldt zur IT. Germanistische Forschung und Lehre in Ungarn: \\ Tendenzen und Perspektiven im Referenzsystem des europäischen Hochschulraums \\ Argumentum 15 (2019), 799-824 \\ Debreceni Egyetemi Kiadó \\ DOI: 10.34103/ARGUMENTUM/2019/6
}

Bild: Die generelle Tendenz zur Senkung der staatlichen Unterstützung von Forschung und Lehre an Universitäten wird in Ungarn weiter verschärft durch deren im Vergleich zu anderen EU-Ländern besonders niedrigen Anteil am Bruttoinlandsprodukt. Selbst innerhalb dieses niedrigen Anteils wird die geisteswissenschaftliche Forschung jedoch stark benachteiligt.

$\mathrm{Zu}$ diesen allgemeinen wissenschaftspolitischen Präferenzen gesellen sich zusätzlich folgende interne wissenschaftssoziologische Eigenheiten der Geisteswissenschaften, die ihre Krise weiter verschärfen:

(e) Ein wichtiges Merkmal der gegenwärtigen Forschungs- und Hochschulpolitik in Ungarn ist es, dass scientometrischen Parametern eine überaus große Bedeutung zugeschrieben wird. Persönliche Karrieren genauso wie die Unterstützung von Projekten hängen in den Naturwissenschaften nicht zuletzt davon ab, über welche scientometrischen Werte eine bestimmte wissenschaftliche Leistung verfügt. Dass es in den Geisteswissenschaften keine konsensfähigen Evaluationsnormen gibt, die ihre wissenschaftlichen Leistungen mit denen der Naturwissenschaften zumindest annähernd vergleichbar machen könnten, trägt ebenfalls dazu bei, dass die Geisteswissenschaften für minderwertig gehalten und sowohl vor der breiten Öffentlichkeit als auch in den Geldgeber-Gremien als solche kommuniziert werden.

(f) Sehr schwach ist auch das Selbstmarketing der Geisteswissenschaftler. Selbst herausragende intellektuelle Leistungen bleiben verborgen und erreichen weder die breite Öffentlichkeit noch die Foren, in denen über künftige Forschungen entschieden wird.

(g) Die Position der Geisteswissenschaften wird auch dadurch geschwächt, dass sie - im Unterschied zu den traditionellen naturwissenschaftlichen Disziplinen wie etwa Physik, Chemie oder Biologie - keine paradigmatischen Disziplinen im Sinne von Kuhn (1970) darstellen, sondern vielmehr kleine, zersplitterte Forschungsbereiche ohne einheitliches Wertesystem, einheitliche Interessen, Methoden und Durchsetzungskraft umfassen. In erster Annäherung scheint dieser Feststellung die Tatsache zu widersprechen, dass etwa ab Mitte des zwanzigsten Jahrhunderts parallel zu andersartigen Entwicklungstendenzen die Umstrukturierung des Systems wissenschaftlicher Disziplinen nicht nur ihre Desintegration, sondern auch ihre Integration bewirkte. Die Integrationsvorgänge betreffen auch die meisten geisteswissenschaftlichen Bereiche, die nicht nur untereinander, sondern auch mit einer Reihe gesellschafts- und sogar naturwissenschaftlicher Disziplinen auf vielfältige Weise effektive interdisziplinäre Kooperationen entwickelten. Man denke dabei nicht nur an die Bindestrichdisziplinen, sondern vor allem an solche wirkungsvollen Strömungen wie etwa die Semiotik, die Kognitionswissenschaften oder in jüngster Zeit die angewandte Philosophie. Allerdings werden diese Integrationsvorgänge, die gewisse Geisteswissenschaften (z. B. Linguistik oder Philosophie) mit naturwissenschaftlichen Forschungen (Biologie, Informatik oder Kognitionswissenschaft) sowie Gesellschaftswissenschaften (Soziologie bzw. Psychologie) im Rahmen zeitgemäß konzipierter gemeinsamer Projekte in Beziehung setzen könnten, durch das institutionelle System stark eingeschränkt, sodass sie sich in der Evaluation wissenschaftlicher Leistungen kaum niederschlagen.

(h) Als illustratives Beispiel für die zwiespältige disziplinäre Lage geisteswissenschaftlicher Forschungen mit - bis jetzt unreflektierten - weitreichenden Konsequenzen sei stellvertretend die gegenwärtige Entwicklung der Linguistik erwähnt. Ein konstitutives Merkmal der Linguistik ist es, dass sich ihr Status aufgrund der disziplinären Struktur der Wissenschaften, die sich im neunzehnten Jahrhundert herausbildete, heute nicht mehr eindeutig definieren lässt. Am einen Ende der Skala finden sich Forschungen, die durch eine enge Verflechtung 


\author{
András Kertész \& Csaba Földes: \\ Von Humboldt zur IT. Germanistische Forschung und Lehre in Ungarn: \\ Tendenzen und Perspektiven im Referenzsystem des europäischen Hochschulraums \\ Argumentum 15 (2019), 799-824 \\ Debreceni Egyetemi Kiadó \\ DOI: 10.34103/ARGUMENTUM/2019/6
}

mit der Informatik und den Ingenieurwissenschaften unter Anwendung modernster Spitzentechnologie - z. B. durch die rapide Entwicklung neuer Subdisziplinen wie der Sprachtechnologie oder der Sprachtherapie - den Anforderungen gegenüber „Innovation“ im oben erläuterten engsten Sinne gerecht werden. Das andere Extrem ist die hermeneutische Methodologie beispielsweise in der Stilistik, der Textlinguistik oder in der linguistischen Poetik. Zwischen den beiden Extremen findet sich eine breite Skala verschiedener Varianten linguistischer Forschungen, die u. a. auch gesellschaftswissenschaftliche Methoden nach dem Muster der Soziologie oder der Psychologie umfassen. Allerdings ist es nicht gelungen, mit einer solchen Differenzierung der Disziplin so umzugehen, dass die neuen Möglichkeiten zur „Innovation“ mit der Verteidigung traditioneller Werte der Geisteswissenschaften verbunden werden. Der Verzicht auf die Letzteren mit der Begründung, dass in gewissen eingeschränkten Fällen bereits auch profitbringende „Innovation“ in erreichbare Nähe gerückt ist, würde automatisch dazu führen, dass das Wesen von Geisteswissenschaften - etwa die Thematisierung des Menschen mit der gesamten Komplexität seiner individuellen, kulturellen, gesellschaftlichen Handlungsfähigkeit - aufgegeben wird. Wie begrüßenswert es auch ist, z. B. eine Disziplin wie die Linguistik in Richtung der „Innovation“ zu lenken und wie wünschenswert es auch wäre, das sich dadurch ergebende neue Potenzial wesentlich gezielter und effektiver zu nutzen, ohne bewusste wissenschaftssoziologische und -politische Reflexion birgt eine solche Schwerpunktverlagerung große Gefahren.

\title{
$4 \quad$ Konsequenzen für die ungarische Germanistik
}

Vor der politisch-gesellschaftlichen Wende gab es in Ungarn nur eine geringe Anzahl von Germanistiklehrstühlen mit nur wenigen Studierenden und Dozenten sowie ziemlich eingeschränkten Forschungsmöglichkeiten. Dann boten sich in der ersten Hälfte der neunziger Jahre früher unvorstellbare Möglichkeiten: die Gründung neuer Institute/Lehrstühle an (neuen) Fachhochschulen und Universitäten, die Vervielfachung der Anzahl der Studierenden und Dozenten, eine relativ gute staatliche Finanzierung und ein reiches Angebot an internationalen Kontakten, Stipendien sowie Drittmitteln. ${ }^{4}$ Nach diesem Aufschwung scheint sich das Blatt erneut gewendet zu haben: Was die ungarische Germanistik in den neunziger Jahren aufgebaut hat, ist nun in Gefahr geraten.

Eine praktische Erfahrung, die Dozenten im Hochschulalltag erleben, ist, dass der BolognaProzess die traditionellen Werte des Germanistikstudiums an Universitäten - zusammen mit der bereits kurz geschilderten Krise des Humboldtschen Hochschulsystems und der Präferenz der wirtschaftsorientierten Hochschulausbildung - radikal infrage stellte. Was benötigt wird, ist in erster Linie nicht mehr die Pflege der deutschen Kultur, nicht die Anwendung zentraler Ergebnisse der Sprach- und Literaturtheorie spezifisch auf die Untersuchung der deutschen Sprache und Literatur, nicht die Vermittlung des kulturellen Erbes zwischen den deutschsprachigen Ländern und Ungarn. Vielmehr wird von den Hochschullehrern erwartet, dass sie praktische Kenntnisse in Wirtschaftsdeutsch, über Fremdenverkehr, über deutschsprachiges Verwaltungsmanagement oder über deutschsprachige Informationstechnologie vermitteln. Diese für die Germanistik schlagartig entstandene Situation erfordert ein radikales Umdenken

4 Eine detailreiche praktisch-aktuelle Übersicht zu Aspekten von Lehre, Forschung und internationalen Beziehungen der einzelnen Germanistikinstitute in Ungarn haben z.B. Masát/Lökös (2017) veröffentlicht. 


\author{
András Kertész \& Csaba Földes: \\ Von Humboldt zur IT. Germanistische Forschung und Lehre in Ungarn: \\ Tendenzen und Perspektiven im Referenzsystem des europäischen Hochschulraums \\ Argumentum 15 (2019), 799-824 \\ Debreceni Egyetemi Kiadó \\ DOI: 10.34103/ARGUMENTUM/2019/6
}

und geht mit der zumindest partiellen Aufgabe intellektueller Werte und der weitgehenden Kommerzialisierung der Germanistenausbildung einher. Dass manche Dozenten diese Umorientierung nur schwer oder überhaupt nicht mittragen konnten oder wollten, überrascht nicht. Hinzu kommt, dass das Volumen der aufgenommenen Germanistikstudenten an allen Hochschuleinrichtungen gesenkt wurde. Dies könnte demnächst den Abbau von Dozentenstellen im Bereich Germanistik nach sich ziehen.

Es wäre sicherlich verfehlt, alle neuen Entwicklungen für schädlich zu halten und die Notwendigkeit der Anpassung an aktuelle gesellschaftliche Ansprüche zu leugnen. Genauso verfehlt wäre es aber auch, auf die neue Situation nicht kritisch zu reagieren und mit der Anerkennung des Neuen gleichzeitig auch wertvolle traditionelle Inhalte über Bord zu werfen.

Um die gegenwärtige Situation der Germanistik auch aus der Perspektive der Studierenden beurteilen zu können, soll im Folgenden über das Ergebnis zweier Umfragen berichtet werden. Es ging in beiden Fällen darum, die Vorstellungen der Studierenden über ihre künftige Laufbahn als Germanistikabsolventen sowohl im Lichte der ihnen bekannten jeweiligen politischen und sozialen Faktoren in Ungarn als auch im Referenzsystem des europäischen Hochschulraumes zu dokumentieren.

\title{
5 Tendenzen in den Einstellungen ungarischer Germanistikstudenten zu ihrer
}

\section{Ausbildung, zum Beruf und zur europäischen Identität}

\subsection{Vorbemerkungen}

Im Jahre 2008 wurde unter Germanistikstudenten der Universität Debrecen eine Umfrage durchgeführt. Die Sprache der Erhebung war Deutsch. Zwecks Feststellung etwaiger Veränderungen in den Meinungsbildern und Motivationsstrukturen der Studierenden im letzten Jahrzehnt wurde 2019 die Umfrage unter identischen Bedingungen - an demselben Institut, mit Studierenden derselben Studiengänge, mit Hilfe des unveränderten Fragebogens wiederholt. 2008 nahmen an der Befragung insgesamt 193 Studierende teil, während 2019 die Zahl der Teilnehmer 118 betrug. 2008 wurden die Daten mit IBM SPSS Statistics ausgewertet, während wir 2019 die Software RStudio Version 1.1.383 nutzten. ${ }^{5}$

Die beiden Umfragen sind insofern repräsentativ als daran praktisch alle immatrikulierten Germanistikstudenten aus sämtlichen Fachsemestern teilnahmen. Unsere nachfolgenden Überlegungen, die auf der Auswertung der beiden Umfragen beruhen, sind allerdings nur mit Vorbehalt und gewissen Einschränkungen interpretierbar. Erstens stehen uns nicht alle Informationen der Umfrage 2008 zur Verfügung. Deshalb können die Auswertungsergebnisse der 2019-er Umfrage nicht bei jedem Item des Fragebogens mit dem entsprechenden 2008-er Befund statistisch verglichen werden. Zweitens mussten wir uns, um zumindest Teile des erwähnten Vergleichs durchführen zu können, nach den Präferenzen der Datenauswertung der ersten Umfrage richten, die unserer Meinung nach nicht differenziert genug gewesen sind. Dennoch denken wir, dass unsere Untersuchung - selbst wenn sich handfeste statistische Zusammenhänge nicht aufdecken lassen und weitreichende Schlussfolgerungen nicht gezogen werden dürfen - trotzt dieser Einschränkungen zu einigen vorsichtigen, jedoch interessanten

Die Daten der Umfrage 2008 sind dem Beitrag von Mezei (2009) entnommen worden. Wir danken Katinka Halász für die Datenerhebung der Umfrage 2019. 


\author{
András Kertész \& Csaba Földes: \\ Von Humboldt zur IT. Germanistische Forschung und Lehre in Ungarn: \\ Tendenzen und Perspektiven im Referenzsystem des europäischen Hochschulraums \\ Argumentum 15 (2019), 799-824 \\ Debreceni Egyetemi Kiadó \\ DOI: 10.34103/ARGUMENTUM/2019/6
}

Hypothesen führen könnte. Dabei wollen wir lediglich einige aufschlussreich erscheinende Befunde hervorheben, ohne eine vollständige und umfangreiche Analyse der Daten anzustreben. Eine mögliche Fortsetzung der Studie könnte sich auf eine wesentlich feinere Datenanalyse der 2019-er Umfrage konzentrieren.

\title{
5.2 Hintergrundfragen
}

Die Umfrage 2008 hat im Hinblick auf den Herkunftsort der Studierenden zum einen gezeigt, dass ein wesentlicher Teil der damaligen Studierenden aus kleineren Ortschaften kam, zum anderen, dass sie sich durch das Germanistikstudium an einer renommierten Universität in einer der größten Städte des Landes bewähren wollten. 61\% der Studierenden kamen aus Städten. Allerdings stammten $36 \%$ zur Zeit der Umfrage in einer Ortschaft mit einer geringeren Einwohnerzahl als 5.000. 30\% kamen aus Kleinstädten mit einer Einwohnerzahl zwischen 5.000 und 20.000, sodass es sich bei insgesamt 66\% um Orte mit weniger als 20.000 Einwohnern handelt. Von den restlichen $34 \%$ lebten $7 \%$ in einer Stadt mit einer Einwohnerzahl zwischen 20.000 und 50.000, 4\% kamen aus Städten mit 50.000 bis 100.000 Einwohnern, $20 \%$ aus Städten mit mehr als 100.000 Einwohnern und 3\% aus der Hauptstadt Budapest. 2019 kamen 30\% der Studierenden aus Dörfern und 70\% aus Städten; 78\% aus der nördlichen Tiefebene und $14 \%$ aus Nordungarn. Somit nahm die Anzahl derer, die aus größeren Ortschaften kommen, in den vergangenen elf Jahren leicht zu, siehe Tabellen 1a, 1b, 1c und $1 \mathrm{~d}$.

\begin{tabular}{|l|l|}
\hline Herkunftsort & Prozent \\
\hline Stadt & $61 \%$ \\
\hline Dorf & $39 \%$ \\
\hline
\end{tabular}

Tabelle 1a.

Umfrage 2019: Herkunftsort der Studierenden

\begin{tabular}{|l|c|}
\hline Einwohnerzahl & Prozent \\
\hline weniger als 5.000 & $36 \%$ \\
\hline $5.000-20.000$ & $30 \%$ \\
\hline $20.000-50.000$ & $7 \%$ \\
\hline $50.000-100.000$ & $4 \%$ \\
\hline über 100.000 & $20 \%$ \\
\hline Budapest (über 2.000.000) & $3 \%$ \\
\hline
\end{tabular}

Tabelle 1c.

Umfrage 2008: Herkunftsort der Studierenden

\begin{tabular}{|l|l|}
\hline Herkunftsort & Prozent \\
\hline Stadt & $70 \%$ \\
\hline Dorf & $30 \%$ \\
\hline
\end{tabular}

Tabelle $1 b$.

Umfrage 2019: Herkunftsort der Studierenden

\begin{tabular}{|l|c|}
\hline Einwohnerzahl & Prozent \\
\hline weniger als 5.000 & $29 \%$ \\
\hline $5.000-20.000$ & $29 \%$ \\
\hline $2.0000-50.000$ & $8 \%$ \\
\hline $50.000-100.000$ & $4 \%$ \\
\hline über 100.000 & $28 \%$ \\
\hline Budapest (über 2.000.000) & $2 \%$ \\
\hline
\end{tabular}

Tabelle 1d.

Umfrage 2019: Herkunftsort der Studierenden

Die Informanten äußerten sich auch zum höchsten Schulabschluss der Eltern. Bemerkenswert ist, dass in der Befragung vor elf Jahren die Gesamtheit der Mütter eine höhere Ausbildung hatte als die Gesamtheit der Väter. In der Umfrage 2019 änderte sich das wesentlich. Dabei ist festzustellen, dass 2019 der Anteil der Familien mit etwas höherem Bildungsgrad relativ zu 2008 leicht gestiegen ist, siehe Tabellen 2a und 2b. 
András Kertész \& Csaba Földes:

Von Humboldt zur IT. Germanistische Forschung und Lehre in Ungarn:

Tendenzen und Perspektiven im Referenzsystem des europäischen Hochschulraums

Argumentum 15 (2019), 799-824

Debreceni Egyetemi Kiadó

DOI: 10.34103/ARGUMENTUM/2019/6

\begin{tabular}{|l|r|c|}
\hline Schulabschluss & Vater & Mutter \\
\hline Grundschule & $15 \%$ & $8 \%$ \\
\hline Facharbeiterschule & $39 \%$ & $10 \%$ \\
\hline Abitur & $29 \%$ & $40 \%$ \\
\hline Fachhochschule & $1 \%$ & $29 \%$ \\
\hline Universität & $16 \%$ & $13 \%$ \\
\hline
\end{tabular}

Tabelle $2 a$.

Umfrage 2008: Höchster Schulabschluss der Eltern

\begin{tabular}{|l|c|c|}
\hline Schulabschluss & Vater & Mutter \\
\hline Grundschule & $3 \%$ & $2 \%$ \\
\hline Facharbeiterschule & $34 \%$ & $19 \%$ \\
\hline Abitur & $37 \%$ & $42 \%$ \\
\hline Fachhochschule & $9 \%$ & $17 \%$ \\
\hline Universität & $18 \%$ & $20 \%$ \\
\hline
\end{tabular}

Tabelle $2 b$.

Umfrage 2019: Höchster Schulabschluss der Eltern

Die nächste Frage bezog sich auf die Motivation für das Germanistikstudium. Es konnten zehn Antwortmöglichkeiten mit Hilfe einer Skala zwischen eins und fünf bewertet werden. Auch mehrere Antworten waren möglich. Dabei bedeutete fünf, dass die jeweilige Antwort ganz, und eins, dass sie überhaupt nicht zutraf. In den Tabellen $3 a$ und $3 b$ sind die entsprechenden Werte zusammengefasst. Diese Tabellen zeigen den Prozentsatz der mit fünf bewerteten Aussagen. Die Abbildungen 1 bis 10 und Tabelle 3c differenzieren die Antworten in der Umfrage 2019. Die Abbildungen 1 bis 10 beziehen sich auf die Zeilen in Tabelle 3b. Die vertikale Achse gibt die Frequenz an, während die horizontale die Werte 1 bis 5 auflistet.

Nach wie vor haben die meisten Befragten ein Germanistikstudium gewählt, weil sie bereits in der Schule Deutsch gelernt haben und/oder ihre Deutschkompetenz vertiefen wollten bzw. einen Beruf ergreifen möchten, der Deutschkenntnisse erfordert. In dem letzteren Fall handelt es sich nicht ausschließlich um Lehramt oder Übersetzung, sondern um ein wesentlich breiteres Spektrum an Beschäftigungsmöglichkeiten.

Dabei sind manche Tendenzen besonders interessant. 2008 stimmten 17\% der Aussage, man habe bereits gute Erfahrungen mit deutschsprachigen Ländern gemacht, völlig zu; 2019 stieg dieser Wert auf 22\%. Der Anteil derjenigen, die sich vom Deutschstudium bessere Berufschancen erhoffen, hat sich ebenfalls vergrößert, vgl. Zeilen 3 und Zeilen 5 in den Tabellen 3a und 3b (2008: 65\% und 2019: 73\%; 2008: 28\% und 2019: 39\%). Allerdings sind auch die Werte in den Zeilen 1 und 2 gestiegen, was auf eine Tendenz hinweist, nach der die Wahl des Studienfachs oft in erster Linie nicht auf einen dezidierten Berufswunsch zurückgeht, sondern sich vielmehr aus den allgemeinen Lebensumständen der Studienbewerber ergibt (etwa: Welche Fachrichtung kann man in der Nähe des Wohnortes studieren).

Betrachtet man nur die Umfrage 2019, so ermöglichen auch der Durchschnitt und die Streuung der Werte interessante Beobachtungen. In Tabelle 3c erhielten die Zeilen 1, 2, 3 und 5 die höchsten Durchschnittswerte. Die Streuung ist in Zeile 9 am höchsten, was den Schluss nahelegt, dass die Möglichkeiten der Studierenden, deutschsprachige Länder zu besuchen, sehr unterschiedlich sind. 
András Kertész \& Csaba Földes:

Von Humboldt zur IT. Germanistische Forschung und Lehre in Ungarn:

Tendenzen und Perspektiven im Referenzsystem des europäischen Hochschulraums

Argumentum 15 (2019), 799-824

Debreceni Egyetemi Kiadó

DOI: 10.34103/ARGUMENTUM/2019/6

\begin{tabular}{|c|l|c|}
\hline & Warum haben Sie ein Germanistikstudium gewählt? & Prozent \\
\hline 1 & In der Schule habe ich Deutsch gelernt. & $45 \%$ \\
\hline 2 & $\begin{array}{l}\text { Ich wollte meine Deutschkenntnisse durch eine Ausbildung an der Universität } \\
\text { vertiefen. }\end{array}$ & $58 \%$ \\
\hline 3 & $\begin{array}{l}\text { Ich möchte einen Beruf wählen, in dem ich meine Deutschkenntnisse } \\
\text { anwenden kann. }\end{array}$ & $65 \%$ \\
\hline 4 & Ich möchte in einem deutschsprachigen Land arbeiten. & $16 \%$ \\
\hline 5 & $\begin{array}{l}\text { Von der Kenntnis der deutschen Sprache verspreche ich mir bessere } \\
\text { Berufschancen und ein höheres Gehalt. }\end{array}$ & $28 \%$ \\
\hline 6 & In meiner Familie spricht man Deutsch. & $4 \%$ \\
\hline 7 & Als Kind hatte ich ein Vorbild/Vorbilder, das/die Deutsch sprach(en). & $5 \%$ \\
\hline 8 & In der Gegend, aus der ich stamme, spricht man viel Deutsch. & $2 \%$ \\
\hline 9 & Ich habe gute Erfahrungen mit deutschsprachigen Ländern/Menschen. & $17 \%$ \\
\hline 10 & $\begin{array}{l}\text { Der Germanistikabschluss gilt als staatlich anerkannte Sprachprüfung in } \\
\text { Deutsch. }\end{array}$ & $3 \%$ \\
\hline 11 & Andere Gründe: & $0 \%$ \\
\hline
\end{tabular}

Tabelle $3 a$.

Umfrage 2008: Motivation für das Germanistikstudium

\begin{tabular}{|l|l|c|}
\hline & Warum haben Sie ein Germanistikstudium gewählt? & Prozent \\
\hline 1 & In der Schule habe ich Deutsch gelernt. & $68 \%$ \\
\hline 2 & $\begin{array}{l}\text { Ich wollte meine Deutschkenntnisse durch eine Ausbildung an der Universität } \\
\text { vertiefen. }\end{array}$ & $66 \%$ \\
\hline 3 & $\begin{array}{l}\text { Ich möchte einen Beruf wählen, in dem ich meine Deutschkenntnisse } \\
\text { anwenden kann. }\end{array}$ & $73 \%$ \\
\hline 4 & Ich möchte in einem deutschsprachigen Land arbeiten. & $17 \%$ \\
\hline 5 & $\begin{array}{l}\text { Von der Kenntnis der deutschen Sprache verspreche ich mir bessere } \\
\text { Berufschancen und ein höheres Gehalt. }\end{array}$ & $39 \%$ \\
\hline 6 & In meiner Familie spricht man Deutsch. & $4 \%$ \\
\hline 7 & Als Kind hatte ich ein Vorbild/Vorbilder, das/die Deutsch sprach(en). & $6 \%$ \\
\hline 8 & In der Gegend, aus der ich stamme, spricht man viel Deutsch. & $1 \%$ \\
\hline 9 & Ich habe gute Erfahrungen mit deutschsprachigen Ländern/Menschen. & $22 \%$ \\
\hline 10 & Der Germanistikabschluss gilt als staatlich anerkannte Sprachprüfung in Deutsch. & $7 \%$ \\
\hline 11 & Andere Gründe: & $0 \%$ \\
\hline
\end{tabular}

Tabelle $3 b$.

Umfrage 2019: Motivation für das Germanistikstudium

\begin{tabular}{|l|l|l|l|}
\hline & Warum haben Sie ein Germanistikstudium gewählt? & Durchschnitt & Streuung \\
\hline 1 & In der Schule habe ich Deutsch gelernt. & 4,49 & 0,88 \\
\hline 2 & $\begin{array}{l}\text { Ich wollte meine Deutschkenntnisse durch eine Ausbildung } \\
\text { an der Universität vertiefen. }\end{array}$ & 4,55 & 0,75 \\
\hline
\end{tabular}


András Kertész \& Csaba Földes:

Von Humboldt zur IT. Germanistische Forschung und Lehre in Ungarn:

Tendenzen und Perspektiven im Referenzsystem des europäischen Hochschulraums

Argumentum 15 (2019), 799-824

Debreceni Egyetemi Kiadó

DOI: 10.34103/ARGUMENTUM/2019/6

\begin{tabular}{|l|l|l|l|}
\hline 3 & $\begin{array}{l}\text { Ich möchte einen Beruf wählen, in dem ich meine } \\
\text { Deutschkenntnisse anwenden kann. }\end{array}$ & 4,63 & 0,7 \\
\hline 4 & Ich möchte in einem deutschsprachigen Land arbeiten. & 3,15 & 1,23 \\
\hline 5 & $\begin{array}{l}\text { Von der Kenntnis der deutschen Sprache verspreche ich mir } \\
\text { bessere Berufschancen und ein höheres Gehalt. }\end{array}$ & 4,13 & 0,88 \\
\hline 6 & In meiner Familie spricht man Deutsch. & 1,66 & 1,19 \\
\hline 7 & $\begin{array}{l}\text { Als Kind hatte ich ein Vorbild/Vorbilder, das/die Deutsch } \\
\text { sprach(en). }\end{array}$ & 1,74 & 1,19 \\
\hline 8 & \begin{tabular}{l} 
In der Gegend, aus der ich stamme, spricht man viel Deutsch. \\
\hline 9
\end{tabular} & $\begin{array}{l}\text { Ich habe gute Erfahrungen mit deutschsprachigen } \\
\text { Ländern/Menschen. }\end{array}$ & 3,08 \\
\hline 10 & $\begin{array}{l}\text { Der Germanistikabschluss gilt als staatlich anerkannte } \\
\text { Sprachprüfung in Deutsch. }\end{array}$ & 2,09 & 1,45 \\
\hline 11 & Andere Gründe: & - & 1,31 \\
\hline
\end{tabular}

Tabelle $3 c$.

Umfrage 2019: Motivation für das Germanistikstudium: Durchschnitt und Streuung

Abbildungen 1 bis 10:

Zeile 1

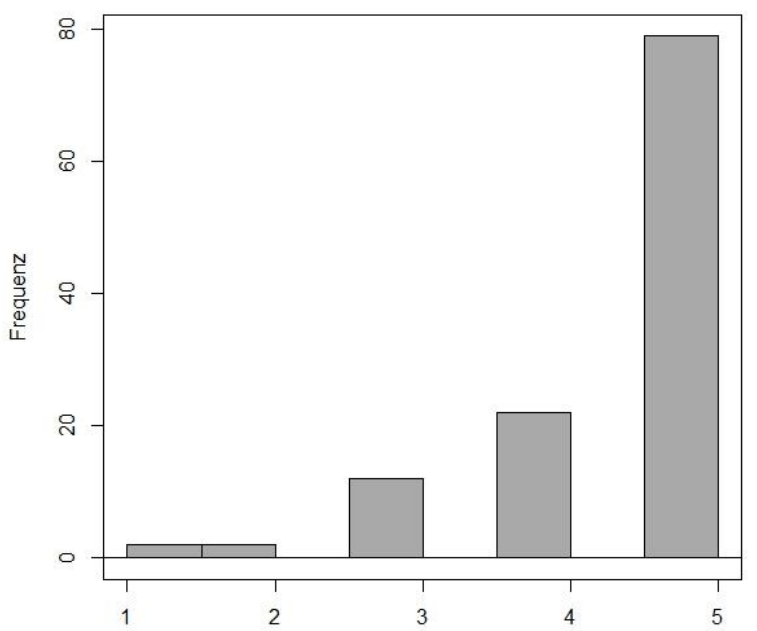

Zeile 2

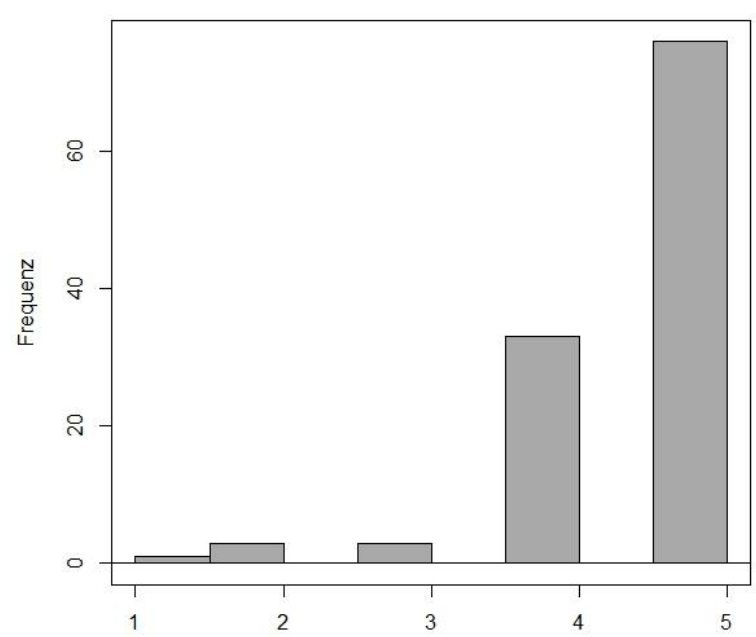


András Kertész \& Csaba Földes:

Von Humboldt zur IT. Germanistische Forschung und Lehre in Ungarn:

Tendenzen und Perspektiven im Referenzsystem des europäischen Hochschulraums

Argumentum 15 (2019), 799-824

Debreceni Egyetemi Kiadó

DOI: 10.34103/ARGUMENTUM/2019/6
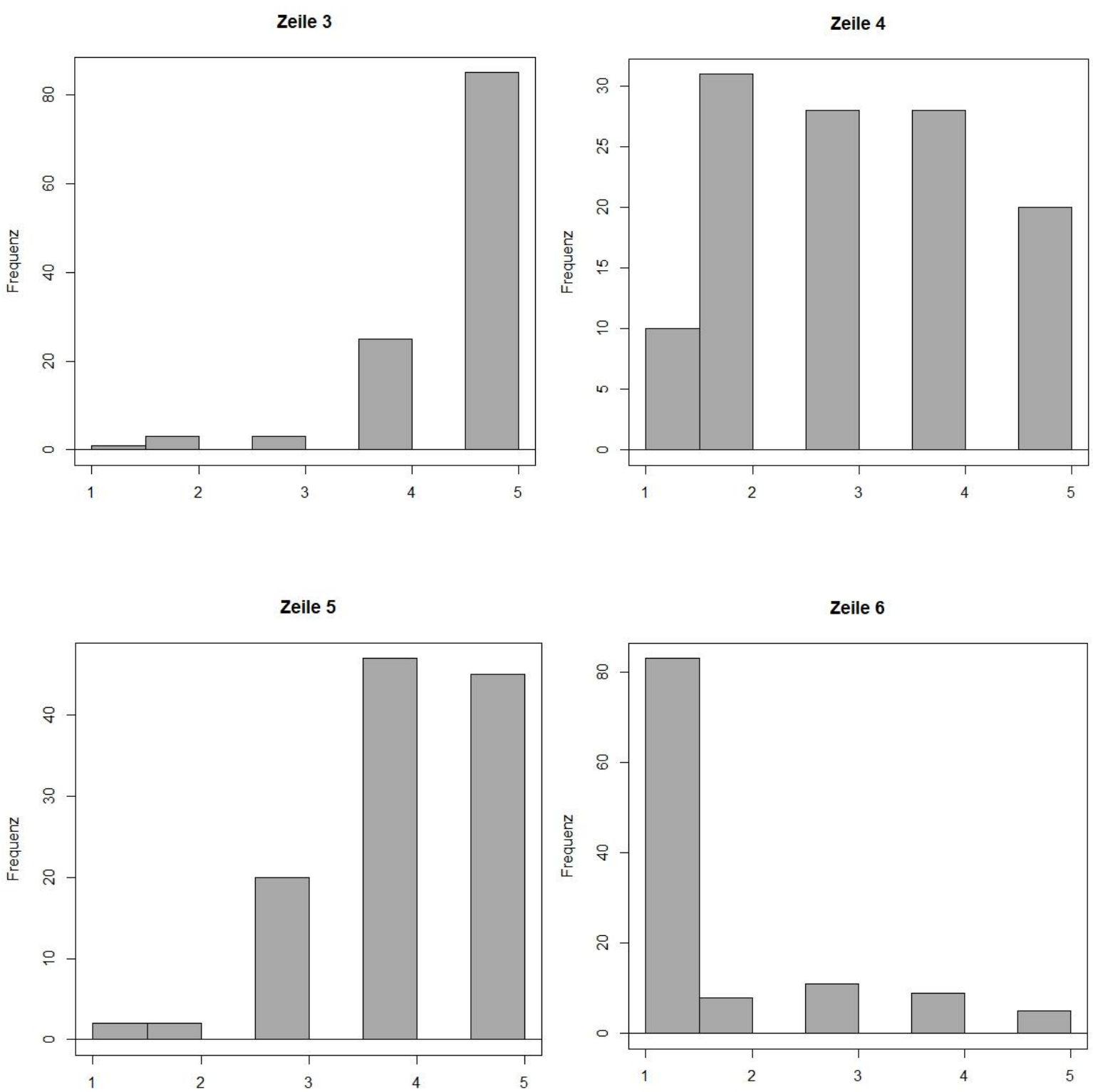

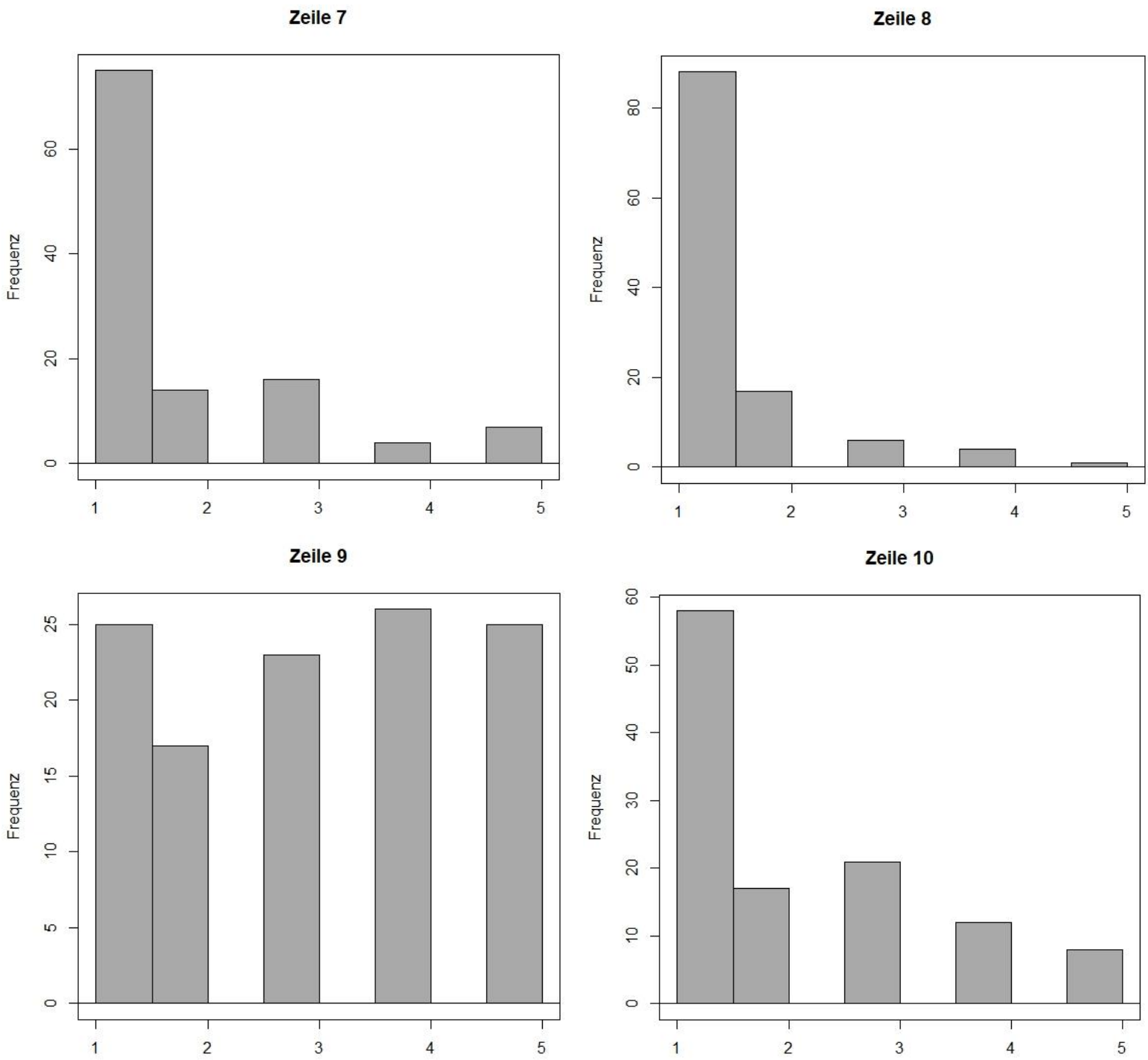

Anschließend wurden die Germanistikstudenten nach ihren beruflichen Plänen befragt. Die Tabellen 4a und 4b zeigen eine deutliche Schwerpunktverlagerung: Zwar blieb der Anteil derer, die Lehrer werden möchten, beinahe konstant, aber 2019 wünschten sich wesentlich weniger Studierende eine Übersetzer- oder Dolmetscherlaufbahn (2008: 72 \% und 2019: 54 \%), während Manager als Wunschberuf im Vergleich zur Umfrage 2008 fast doppelt so oft auftrat (2008: $17 \%$ und 2019: 32 \%). In der Zeile „Andere“ (7\%) haben die meisten den IT-Bereich angegeben.

\begin{tabular}{|l|c|}
\hline Angestrebter Beruf & Prozent \\
\hline Lehrer & $55 \%$ \\
\hline Manager & $17 \%$ \\
\hline Übersetzer/Dolmetscher & $72 \%$ \\
\hline Fremdenführer & $45 \%$ \\
\hline Ein Beruf im & $5 \%$ \\
\hline
\end{tabular}

\begin{tabular}{|l|c|}
\hline Angestrebter Beruf & Prozent \\
\hline Lehrer & $50 \%$ \\
\hline Manager & $32 \%$ \\
\hline Übersetzer/Dolmetscher & $54 \%$ \\
\hline Fremdenführer & $31 \%$ \\
\hline Ein Beruf im & $6 \%$ \\
\hline
\end{tabular}


András Kertész \& Csaba Földes:

Von Humboldt zur IT. Germanistische Forschung und Lehre in Ungarn:

Tendenzen und Perspektiven im Referenzsystem des europäischen Hochschulraums

Argumentum 15 (2019), 799-824

Debreceni Egyetemi Kiadó

DOI: 10.34103/ARGUMENTUM/2019/6

\begin{tabular}{|l|l|}
\hline Wirtschaftssektor & \\
\hline Ein Beruf in der Verwaltung & $2 \%$ \\
\hline Andere & $3 \%$ \\
\hline
\end{tabular}

Tabelle $4 a$.

Umfrage 2008: Angestrebter Beruf

\begin{tabular}{|l|l|}
\hline Wirtschaftssektor & \\
\hline Ein Beruf in der Verwaltung & $0 \%$ \\
\hline Andere & $7 \%$ \\
\hline
\end{tabular}

Tabelle $4 b$.

Umfrage 2019: Angestrebter Beruf

\subsection{Angaben zum Studium und zu den Sprachkenntnissen}

\subsubsection{Sprachfertigkeiten}

Aus der Tabelle 5 geht hervor, dass 2019 fast zwei Drittel der Studierenden (63\%) seit mindestens 10 Jahren Deutsch lernen. Die entsprechenden Daten aus dem Jahr 2008 waren uns leider nicht zugänglich.

\begin{tabular}{|l|c|}
\hline $\begin{array}{l}\text { Wie lange lernen Sie bereits } \\
\text { Deutsch? }\end{array}$ & Prozent \\
\hline 1-2 Jahre & $1 \%$ \\
\hline 3-4 Jahre & $6 \%$ \\
\hline 5-6 Jahre & $14 \%$ \\
\hline 7-8 Jahre & $16 \%$ \\
\hline 9-10 Jahre & $17 \%$ \\
\hline länger als 10 Jahre & $46 \%$ \\
\hline
\end{tabular}

Tabelle 5.

Umfrage 2019: Wie lange lernen Sie bereits Deutsch?

Die nächste Frage bezog sich auf die erachtete Wichtigkeit von Kenntnissen europäischer Fremdsprachen für das Studium. Bei der Beantwortung dieser Frage bedeutete eins, dass die jeweilige Sprache überhaupt nicht wichtig und fünf, dass sie sehr wichtig ist. Die Tabellen 6a und $6 \mathrm{~b}$ zeigen, dass die Anzahl derer, die sich wegen der Lernmöglichkeit des Deutschen für das Fach Germanistik entscheiden, im letzten Jahrzehnt anstieg (2008: 85 \% und 2019: 93 \%).

\begin{tabular}{|l|l|l|}
\hline & Sprachen & sehr wichtig \\
\hline 1 & Deutsch & $85 \%$ \\
\hline 2 & Englisch & $39 \%$ \\
\hline 3 & Französisch & $2 \%$ \\
\hline 4 & Russisch & $3 \%$ \\
\hline 5 & Spanisch & $1 \%$ \\
\hline 6 & Italienisch & $1 \%$ \\
\hline 7 & Niederländisch & $9 \%$ \\
\hline
\end{tabular}

Tabelle $6 a$.

Umfrage 2008: Wichtigkeit der

europäischen Fremdsprachen für das

Studium der Germanistikstudenten

\begin{tabular}{|l|l|c|}
\hline & Sprachen & sehr wichtig \\
\hline 1 & Deutsch & $93 \%$ \\
\hline 2 & Englisch & $40 \%$ \\
\hline 3 & Französisch & $1 \%$ \\
\hline 4 & Russisch & $4 \%$ \\
\hline 5 & Spanisch & $0 \%$ \\
\hline 6 & Italienisch & $2 \%$ \\
\hline 7 & Niederländisch & $7 \%$ \\
\hline
\end{tabular}

\section{Tabelle $6 b$}

Umfrage 2019: Wichtigkeit der

europäischen Fremdsprachen für das

Studium der Germanistikstudenten 
András Kertész \& Csaba Földes:

Von Humboldt zur IT. Germanistische Forschung und Lehre in Ungarn:

Tendenzen und Perspektiven im Referenzsystem des europäischen Hochschulraums

Argumentum 15 (2019), 799-824

Debreceni Egyetemi Kiadó

DOI: 10.34103/ARGUMENTUM/2019/6

Bei den Tabellen $7 \mathrm{a}$ und $7 \mathrm{~b}$, in denen es um die Bedeutung der Fremdsprachen für das zukünftige Berufsleben der Studierenden geht, fällt auf, dass 2019 die berufliche Relevanz des Deutschen höher (2008: 77 \%, 2019: 92 \%) und die des Englischen niedriger (2008: 57 \%, 2013: 48 \%) eingeschätzt wurde als elf Jahre zuvor.

\begin{tabular}{|c|l|c|}
\hline & Sprache & sehr wichtig \\
\hline 1 & Deutsch & $77 \%$ \\
\hline 2 & Englisch & $57 \%$ \\
\hline 3 & Französisch & $4 \%$ \\
\hline 4 & Russisch & $2 \%$ \\
\hline 5 & Spanisch & $3 \%$ \\
\hline 6 & Italienisch & $3 \%$ \\
\hline 7 & Niederländisch & $9 \%$ \\
\hline
\end{tabular}

Tabelle $7 a$.

Umfrage 2008: Wichtigkeit der europäischen Fremdsprachen für den zukünftigen Beruf von Germanistikstudenten

\begin{tabular}{|c|l|c|}
\hline & Sprache & sehr wichtig \\
\hline 1 & Deutsch & $92 \%$ \\
\hline 2 & Englisch & $48 \%$ \\
\hline 3 & Französisch & $1 \%$ \\
\hline 4 & Russisch & $5 \%$ \\
\hline 5 & Spanisch & $0 \%$ \\
\hline 6 & Italienisch & $3 \%$ \\
\hline 7 & Niederländisch & $2 \%$ \\
\hline
\end{tabular}

Tabelle $7 b$.

Umfrage 2019: Wichtigkeit der europäischen Fremdsprachen für den zukünftigen Beruf von Germanistikstudenten

Auf die Frage, ob die Befragten ihre Deutschkenntnisse auch außerhalb des Studiums nutzten, stehen uns die Antworten aus dem Jahr 2008 nicht zur Verfügung, 2019 haben 88\% diese Frage mit , ja“ beantwortet. Über die jeweiligen Tätigkeiten gibt Tabelle 8 Aufschluss (wobei mehr als eine Antwort möglich war). In der Zeile „Andere Gründe“ (10 \%) waren Angaben wie die Möglichkeit der Erteilung von Privatunterricht in Deutsch sowie das Hören von deutscher Musik am häufigsten.

\begin{tabular}{|c|l|c|}
\hline & Wofür verwenden Sie Deutsch außerhalb des Studiums? & Prozent \\
\hline 1 & Ich sehe deutschsprachige Fernsehsendungen & $72 \%$ \\
\hline 2 & Ich lese deutschsprachige Zeitungen/Magazine/Bücher usw. & $81 \%$ \\
\hline 3 & Korrespondenz mit Menschen, die Deutsch als Muttersprache haben & $34 \%$ \\
\hline 4 & Korrespondenz mit Menschen, die Deutsch als Fremdsprache beherrschen & $21 \%$ \\
\hline 5 & Private Kontakte innerhalb der engen Familie & $6 \%$ \\
\hline 6 & Private Kontakte innerhalb der ferneren Verwandtschaft & $13 \%$ \\
\hline 7 & Kontakte zu deutschsprachigen Freunden/Bekannten & $46 \%$ \\
\hline 8 & Kontakte zu Freunden/Bekannten, deren Muttersprache nicht Deutsch ist & $27 \%$ \\
\hline 9 & Freizeitbeschäftigungen mit deutschsprachigen Freunden/Bekannten & $14 \%$ \\
\hline 10 & $\begin{array}{l}\text { Freizeitbeschäftigungen mit Menschen, die Deutsch als Fremdsprache } \\
\text { beherrschen }\end{array}$ & $19 \%$ \\
\hline 11 & Surfen auf deutschsprachigen Webseiten & $67 \%$ \\
\hline 12 & Andere Gründe: & $10 \%$ \\
\hline
\end{tabular}

Tabelle 8 .

Verwendung des Deutschen außerhalb des Studiums 
András Kertész \& Csaba Földes:

Von Humboldt zur IT. Germanistische Forschung und Lehre in Ungarn:

Tendenzen und Perspektiven im Referenzsystem des europäischen Hochschulraums

Argumentum 15 (2019), 799-824

Debreceni Egyetemi Kiadó

DOI: 10.34103/ARGUMENTUM/2019/6

\subsubsection{Einstellung zur Mehrsprachigkeit}

Mehrsprachigkeit, d.h. der Erwerb und die Beherrschung von mehr als einer Sprache sowie die Realität, dass in Europa viele Sprachen gesprochen werden und die europäische Politik die Sprachenvielfalt mit unterschiedlichen Mitteln fördert, ist einer der wichtigsten Werte der europäischen Identität. Daher ist zu bemerken, dass im Jahr 2008 rund 90 \% der Studierenden Mehrsprachigkeit für sehr wichtig oder für wichtig hielten, wobei 2019 diese Zahl leicht - auf $94 \%$ - gestiegen ist.

\begin{tabular}{|l|c|}
\hline Grad der Wichtigkeit & Prozent \\
\hline Äußerst wichtig & $49 \%$ \\
\hline Wichtig & $43 \%$ \\
\hline Neutral & $5 \%$ \\
\hline Nicht besonders wichtig & $1 \%$ \\
\hline Völlig unwichtig & $2 \%$ \\
\hline
\end{tabular}

Tabelle 9 a.

Umfrage 2008: Einstellung der Germanistikstudenten zu einer allgemeinen mehrsprachigen Ausbildung

\begin{tabular}{|l|c|}
\hline Grad der Wichtigkeit & Prozent \\
\hline Äußerst wichtig & $53 \%$ \\
\hline Wichtig & $41 \%$ \\
\hline Neutral & $4 \%$ \\
\hline Nicht besonders wichtig & $1 \%$ \\
\hline Völlig unwichtig & $1 \%$ \\
\hline
\end{tabular}

Tabelle $9 b$.

Umfrage 2019: Einstellung der Germanistikstudenten zu einer allgemeinen mehrsprachigen Ausbildung

Die Zahl derer, die der Meinung sind, dass Mehrsprachigkeit zur Herausbildung bzw. zur Stärkung einer europäischen Identität beiträgt, vergrößerte sich 2019 im Vergleich zu 2008 etwas (2008: 68\%, 2019: $75 \%$ ), siehe Tabellen 10a und 10b.

\begin{tabular}{|l|c|}
\hline $\begin{array}{l}\text { Mehrsprachigkeit } \\
\text { trägt zur Herausbildung } \\
\text { einer europäischen Identität } \\
\text { bei }\end{array}$ & Prozent \\
\hline Ich stimme völlig zu. & $30 \%$ \\
\hline Ich stimme teilweise zu. & $38 \%$ \\
\hline Ich stimme teilweise nicht zu. & $25 \%$ \\
\hline Ich stimme nicht zu. & $7 \%$ \\
\hline Ich weiß es nicht. & $0 \%$ \\
\hline
\end{tabular}

Tabelle 10a.

Umfrage 2008: Mehrsprachigkeit trägt zur Herausbildung einer europäischen Identität bei

\begin{tabular}{|l|c|}
\hline $\begin{array}{l}\text { Mehrsprachigkeit } \\
\text { trägt zur Herausbildung } \\
\text { einer europäischen Identität } \\
\text { bei }\end{array}$ & Prozent \\
\hline Ich stimme völlig zu. & $30 \%$ \\
\hline Ich stimme teilweise zu. & $45 \%$ \\
\hline Ich stimme teilweise nicht zu. & $20 \%$ \\
\hline Ich stimme nicht zu. & $4 \%$ \\
\hline Keine Stellungnahme. & $1 \%$ \\
\hline
\end{tabular}

\section{Tabelle $10 b$.}

Umfrage 2019: Mehrsprachigkeit trägt zur Herausbildung einer europäischen Identität bei

Während 2008 insgesamt $69 \%$ der Studierenden voll oder teilweise damit einverstanden waren, dass Mehrsprachigkeit zur Herausbildung bzw. Stärkung einer europäischen Identität beitrage, stimmten 2019 dieser Behauptung schon 86 \% völlig oder teilweise zu. 
András Kertész \& Csaba Földes:

Von Humboldt zur IT. Germanistische Forschung und Lehre in Ungarn:

Tendenzen und Perspektiven im Referenzsystem des europäischen Hochschulraums

Argumentum 15 (2019), 799-824

Debreceni Egyetemi Kiadó

DOI: 10.34103/ARGUMENTUM/2019/6

\begin{tabular}{|l|c|}
\hline $\begin{array}{l}\text { Mehrsprachigkeit fördert die } \\
\text { Vielfalt von Forschung und } \\
\text { Lehre }\end{array}$ & Prozent \\
\hline Ich stimme völlig zu. & $34 \%$ \\
\hline Ich stimme teilweise zu. & $35 \%$ \\
\hline Ich stimme teilweise nicht zu. & $24 \%$ \\
\hline Ich stimme nicht zu. & $4 \%$ \\
\hline Keine Stellungnahme. & $2 \%$ \\
\hline
\end{tabular}

Tabelle 11a.

Umfrage 2008: Mehrsprachigkeit fördert

die Vielfalt von Forschung und Lehre

\begin{tabular}{|l|c|}
\hline $\begin{array}{l}\text { Mehrsprachigkeit fördert die } \\
\text { Vielfalt von Forschung und } \\
\text { Lehre. }\end{array}$ & Prozent \\
\hline Ich stimme völlig zu. & $46 \%$ \\
\hline Ich stimme teilweise zu. & $41 \%$ \\
\hline Ich stimme teilweise nicht zu. & $11 \%$ \\
\hline Ich stimme nicht zu. & $2 \%$ \\
\hline Keine Stellungnahme & $0 \%$ \\
\hline
\end{tabular}

Tabelle $11 \mathrm{~b}$

Umfrage 2019: Mehrsprachigkeit fördert

die Vielfalt von Forschung und Lehre

Die Beurteilung der Rolle des Englischen hat sich in den vergangenen 11 Jahren ebenfalls deutlich verändert: 2008 stimmten insgesamt $37 \%$ der Befragten völlig oder teilweise zu, dass Englisch in der Bildung ausreicht. 2019 beträgt der Anteil $50 \%$, siehe Tabellen 12a und 12b.

\begin{tabular}{|l|l|}
\hline $\begin{array}{l}\text { Englisch als ,Weltsprache“6 } \\
\text { reicht in der Bildung völlig } \\
\text { aus }\end{array}$ & Prozent \\
\hline Ich stimme völlig zu. & $14 \%$ \\
\hline Ich stimme teilweise zu. & $23 \%$ \\
\hline Ich stimme teilweise nicht zu. & $21 \%$ \\
\hline Ich stimme nicht zu. & $27 \%$ \\
\hline Keine Stellungnahme & $15 \%$ \\
\hline
\end{tabular}

Tabelle $12 a$.

Umfrage 2008: Englisch als ,, Weltsprache“ reicht in der Bildung völlig aus.

\begin{tabular}{|l|l|}
\hline $\begin{array}{l}\text { Englisch als „Weltsprache“ } \\
\text { reicht in der Bildung völlig } \\
\text { aus }\end{array}$ & Prozent \\
\hline Ich stimme völlig zu. & $24 \%$ \\
\hline Ich stimme teilweise zu. & $26 \%$ \\
\hline Ich stimme teilweise nicht zu. & $17 \%$ \\
\hline Ich stimme nicht zu. & $20 \%$ \\
\hline Keine Stellungnahme & $12 \%$ \\
\hline
\end{tabular}

Tabelle $12 b$

Umfrage 2019: Englisch als ,Weltsprache“ reicht in der Bildung völlig aus.

Auch die Antworten auf die nächste Frage scheinen mit dieser Tendenz im Einklang zu stehen: 2008 stimmten $38 \%$ der Studierenden der Aussage völlig oder teilweise zu, dass Ungarisch und Englisch für das Berufsleben ausreichen, 2019 wuchs dieser Prozentsatz auf 52 $\%$, siehe Tabellen 13a und $13 \mathrm{~b}$.

\begin{tabular}{|l|l|}
\hline $\begin{array}{l}\text { Ungarisch und Englisch } \\
\text { reichen für das Berufsleben } \\
\text { aus }\end{array}$ & Prozent \\
\hline Ich stimme völlig zu. & $13 \%$ \\
\hline Ich stimme teilweise zu. & $25 \%$ \\
\hline Ich stimme teilweise nicht zu. & $26 \%$ \\
\hline Ich stimme nicht zu. & $23 \%$ \\
\hline Keine Stellungnahme & $13 \%$ \\
\hline
\end{tabular}

Tabelle $13 a$.

Umfrage 2008: Ungarisch und Englisch reichen für das Berufsleben aus

\begin{tabular}{|l|l|}
\hline $\begin{array}{l}\text { Ungarisch und Englisch } \\
\text { reichen für das Berufsleben } \\
\text { aus }\end{array}$ & Prozent \\
\hline Ich stimme völlig zu. & $27 \%$ \\
\hline Ich stimme teilweise zu. & $26 \%$ \\
\hline Ich stimme teilweise nicht zu. & $17 \%$ \\
\hline Ich stimme nicht zu. & $22 \%$ \\
\hline Keine Stellungnahme & $8 \%$ \\
\hline
\end{tabular}

Tabelle $13 b$.

Umfrage 2019: Ungarisch und Englisch reichen für das Berufsleben aus 
András Kertész \& Csaba Földes:

Von Humboldt zur IT. Germanistische Forschung und Lehre in Ungarn:

Tendenzen und Perspektiven im Referenzsystem des europäischen Hochschulraums

Argumentum 15 (2019), 799-824

Debreceni Egyetemi Kiadó

DOI: 10.34103/ARGUMENTUM/2019/6

Demgegenüber hat sich der Anteil derer, die gewillt wären, weitere Sprachen zu lernen, falls sie mehr Zeit hätten, nicht grundsätzlich geändert (2008: $80 \%$ und 2019: $82 \%$ ), siehe Tabellen 14a und 14b.

\begin{tabular}{|l|c|}
\hline $\begin{array}{l}\text { Alle Sprachen sind wichtig } \\
\text { und wenn ich mehr Zeit hätte, } \\
\text { würde ich mehr Sprachen } \\
\text { lernen }\end{array}$ & Prozent \\
\hline Ich stimme völlig zu. & $57 \%$ \\
\hline Ich stimme teilweise zu. & $23 \%$ \\
\hline Ich stimme teilweise nicht zu. & $12 \%$ \\
\hline Ich stimme nicht zu. & $6 \%$ \\
\hline Keine Stellungnahme & $2 \%$ \\
\hline
\end{tabular}

Tabelle 14a.

Umfrage 2008: Alle Sprachen sind wichtig und wenn ich mehr Zeit hätte, würde ich mehr Sprachen lernen

\begin{tabular}{|l|c|}
\hline $\begin{array}{l}\text { Alle Sprachen sind wichtig } \\
\text { und wenn ich mehr Zeit hätte, } \\
\text { würde ich mehr Sprachen } \\
\text { lernen }\end{array}$ & Prozent \\
\hline Ich stimme völlig zu. & $56 \%$ \\
\hline Ich stimme teilweise zu. & $26 \%$ \\
\hline Ich stimme teilweise nicht zu. & $9 \%$ \\
\hline Ich stimme nicht zu. & $9 \%$ \\
\hline Keine Stellungnahme & $1 \%$ \\
\hline
\end{tabular}

\section{Tabelle $14 b$.}

Umfrage 2019: Alle Sprachen sind wichtig und wenn ich mehr Zeit hätte, würde ich mehr Sprachen lernen

Die nächste Frage bezieht sich auf einen häufig artikulierten möglichen Nachteil der Mehrsprachigkeit. 2008 stimmten insgesamt $29 \%$ der Informanten völlig oder teilweise zu, dass Mehrsprachigkeit auch Missverständnisse verursachen könne. 2019 liegt der entsprechende Prozentsatz bei $37 \%$.

\begin{tabular}{|l|c|}
\hline $\begin{array}{l}\text { Durch Mehrsprachigkeit } \\
\text { häufen sich die } \\
\text { Missverständnisse }\end{array}$ & Prozent \\
\hline Ich stimme völlig zu. & $5 \%$ \\
\hline Ich stimme teilweise zu. & $24 \%$ \\
\hline Ich stimme teilweise nicht zu. & $39 \%$ \\
\hline Ich stimme nicht zu. & $19 \%$ \\
\hline Keine Stellungnahme & $13 \%$ \\
\hline
\end{tabular}

Tabelle $15 a$

Umfrage 2008: Durch Mehrsprachigkeit

häufen sich die Missverständnisse

\begin{tabular}{|l|c|}
\hline $\begin{array}{l}\text { Durch Mehrsprachigkeit } \\
\text { häufen sich die } \\
\text { Missverständnisse }\end{array}$ & Prozent \\
\hline Ich stimme völlig zu. & $9 \%$ \\
\hline Ich stimme teilweise zu. & $28 \%$ \\
\hline Ich stimme teilweise nicht zu. & $36 \%$ \\
\hline Ich stimme nicht zu. & $21 \%$ \\
\hline Keine Stellungnahme & $5 \%$ \\
\hline
\end{tabular}

Tabelle $15 b$

Umfrage 2019: Durch Mehrsprachigkeit

häufen sich die Missverständnisse

\subsubsection{Die Rolle der deutschen Sprachpraxis für Studium und Beruf}

Aus der Frage, was die Studierenden von einem Auslandssemester hielten, ging hervor, dass $54 \%$ der Studierenden ein Gaststudium in einem deutschsprachigen Land planten.

\begin{tabular}{|l|l|l|}
\hline & Gründe für ein Austauschstudium & Prozent \\
\hline 1 & $\begin{array}{l}\text { Weil mein Fachbereich ein Austauschprogramm mit der } \\
\text { Zieluniversität hat. }\end{array}$ & $27 \%$ \\
\hline 2 & Weil ich Auslandserfahrung sammeln will. & $64 \%$ \\
\hline 3 & Weil ich Deutsch lernen will. & $84 \%$ \\
\hline
\end{tabular}


András Kertész \& Csaba Földes:

Von Humboldt zur IT. Germanistische Forschung und Lehre in Ungarn:

Tendenzen und Perspektiven im Referenzsystem des europäischen Hochschulraums

Argumentum 15 (2019), 799-824

Debreceni Egyetemi Kiadó

DOI: 10.34103/ARGUMENTUM/2019/6

\begin{tabular}{|c|l|c|}
\hline 4 & $\begin{array}{l}\text { Weil ich mir durch meine im Austauschstudium erworbenen } \\
\text { Deutschkenntnisse bessere Berufschancen verspreche. }\end{array}$ & $58 \%$ \\
\hline 5 & $\begin{array}{l}\text { Weil ich mir durch meine im Austauschstudium erworbenen } \\
\text { Fachkenntnisse bessere Berufschancen verspreche. }\end{array}$ & $46 \%$ \\
\hline 6 & $\begin{array}{l}\text { Weil ich mir durch meine im Austauschstudium erworbenen } \\
\text { Kenntnisse der deutschsprachigen Kultur bessere } \\
\text { Berufschancen verspreche. }\end{array}$ & $40 \%$ \\
\hline 7 & Aus einem anderen Grund: & $0 \%$ \\
\hline
\end{tabular}

Tabelle 16

Umfrage 2019: Gründe für ein Austauschstudium

Das wichtigste Argument gegen ein Austauschstudium ist (Tabelle 17), dass sich dadurch das Studium verlängert. An zweiter und dritter Stelle stehen mangelndes Interesse bzw. finanzielle Gründe. Es ist bemerkenswert, dass in Tabelle 16 die Absicht, Deutsch zu lernen, den höchsten und das Motiv, die deutsche Kultur kennenzulernen, den zweitniedrigsten Wert erhielten.

\begin{tabular}{|l|c|}
\hline Gründe gegen ein Austauschstudium & Prozent \\
\hline Ich habe/hatte keine Möglichkeit. & $5 \%$ \\
\hline Ich interessiere mich dafür nicht. & $24 \%$ \\
\hline Ich will mein Studium dadurch nicht verlängern. & $48 \%$ \\
\hline Aus finanziellen Gründen. & $21 \%$ \\
\hline Meine Fremdsprachenkenntnisse sind dafür zu gering. & $0 \%$ \\
\hline Andere & $2 \%$ \\
\hline
\end{tabular}

Tabelle 17.

Umfrage 2019: Gründe gegen ein Austauschstudium

Zur Beurteilung der Bedeutung des Deutschen im Berufsleben können wir uns nur auf die Daten der Umfrage 2019 stützen. Wie man sieht, hielt die überwiegende Mehrheit der Befragten (insgesamt $87 \%$ ) es für wahrscheinlich oder sehr wahrscheinlich, dass die Bedeutung des Deutschen im Berufsleben steigen wird. Bei dieser Frage hatten die Informanten auch die Möglichkeit, ihrer Wahl Kommentare hinzuzufügen. Viele haben hier ausgeführt, dass sich in Ungarn die Zahl internationaler Unternehmen erhöhen wird, wodurch sich die deutsch-ungarischen Beziehungen vertiefen sowie dass in Mitteleuropa der Einfluss des Deutschen zunehmen wird.

\begin{tabular}{|l|l|}
\hline Die Bedeutung des Deutschen im Berufsleben & Prozent \\
\hline Sie steigt sehr wahrscheinlich. & $53 \%$ \\
\hline Sie steigt wahrscheinlich. & $34 \%$ \\
\hline Sie bleibt vermutlich wie sie ist. & $4 \%$ \\
\hline Sie sinkt wahrscheinlich. & $3 \%$ \\
\hline Sie sinkt sehr wahrscheinlich. & $0 \%$ \\
\hline Keine Stellungnahme & $6 \%$ \\
\hline
\end{tabular}

Tabelle 18.

Umfrage 2019: Die Bedeutung des Deutschen im Berufsleben 
András Kertész \& Csaba Földes:

Von Humboldt zur IT. Germanistische Forschung und Lehre in Ungarn:

Tendenzen und Perspektiven im Referenzsystem des europäischen Hochschulraums

Argumentum 15 (2019), 799-824

Debreceni Egyetemi Kiadó

DOI: 10.34103/ARGUMENTUM/2019/6

\subsection{Auswertung}

\subsubsection{Vorbemerkung}

Im Folgenden seien, ohne eine vollständige Datenanalyse anzustreben, einige uns als relevant erscheinende nicht-triviale Tendenzen hervorgehoben, die den Weg für die Schlussfolgerungen des Beitrags vorbereiten sollen. $\mathrm{Zu}$ diesem Zweck werden unsere Überlegungen nach Kriterien dargestellt, die es ermöglichen, Zusammenhänge unter den Daten zu finden, die in unterschiedlichen Teilen des Fragebogens erfragt worden sind. Somit wird hier die Struktur des Fragebogens nicht wiederholt.

\subsubsection{Einfluss der lokalen wirtschaftlichen und sozialen Bedingungen auf die Stellungnahmen der Studierenden}

Die Antworten auf die Hintergrundfragen zeigen leichte Veränderungen im sozialen Umfeld der Studierenden. Während sie 2008 überwiegend aus kleineren Ortschaften und aus Familien mit niedrigerem Bildungsgrad kamen, nahm 2019 der Anteil derjenigen zu, die aus etwas größeren Ortschaften stammen und deren Väter, aber auch beide Elternteile, einen höheren Bildungsgrad aufwiesen als die der Informanten der 2008-er Umfrage. Allerdings ist die Anzahl der Studierenden, die die Möglichkeit hatten, deutschsprachige Länder kennenzulernen, nach wie vor recht klein.

$\mathrm{Zu}$ den lokalen Bedingungen, die die Informanten-Antworten zu beeinflussen scheinen, gehört die Tatsache, dass in Debrecen mehrere deutsche Großunternehmen angesiedelt sind, die eine große Anziehungskraft auf potenzielle Arbeitnehmer ausüben. Sie bieten gegenwärtig zahlreiche Stellen an, wobei die Anzahl der Absolventen zur Besetzung dieser Stellen bei weitem nicht ausreicht. Beispielsweise wirbt die ungarische Tochterfirma der Deutschen Telekom ständig um die Gunst der Germanistik-Absolventen und auch das mit sehr bedeutender wirtschaftspolitischer Unterstützung gegründete neue BMW-Werk bietet für die nächsten Jahrzehnte gute Berufsmöglichkeiten für Deutschsprechende. Dieser Umstand scheint mit den Daten in den Tabellen 4a und 4b zu korrelieren, denen zufolge 2019 fast doppelt so viele Studierende Manager als Wunschberuf angaben wie noch 2008. Wie die Tabellen $7 \mathrm{a}$ und $7 \mathrm{~b}$ bezeugen, wurde 2019 die Bedeutung des Deutschen für den zukünftigen Beruf höher eingeschätzt als 2008 (2008: 77 \% und 2019: 92 \%). Dies mag zwar mehrere Gründe haben, einer unter ihnen könnte jedoch sein, dass in Debrecen, wie bereits erwähnt, die Nachfrage nach deutschsprechenden Absolventen sehr groß ist. Auch die Antworten auf die Fragen in Tabelle 16 weisen darauf hin, dass die Denkweise der Informanten grundsätzlich nicht durch emotionale oder akademische Faktoren geprägt ist, sondern eher durch praktische Ziele.

Die Tatsache, dass laut Tabellen $6 \mathrm{a}$ und $6 \mathrm{~b}$ die Relevanz des Niederländischen höher bewertet wurde als die des Französischen, Russischen, Spanischen und Italienischen, hat vermutlich damit zu tun, dass die Universität Debrecen auch einen Studiengang Niederlandistik bietet, dessen Popularität die der Fächer Französistik, Italianistik und Slawistik übertrifft (2008: 9 \% und 2019: 7 \%); eine Studienrichtung Hispanistik gibt es nicht. Im Gegensatz zum Aspekt Studium hat sich im Hinblick auf den zukünftigen Beruf der Informanten anscheinend die Bewertung des Niederländischen im Laufe des vergangenen Jahrzehnts deutlich verändert: Im Unterschied zu 2008 (9\%; siehe Tabelle 7a) waren 2019 nur noch $2 \%$ der Meinung, dass 


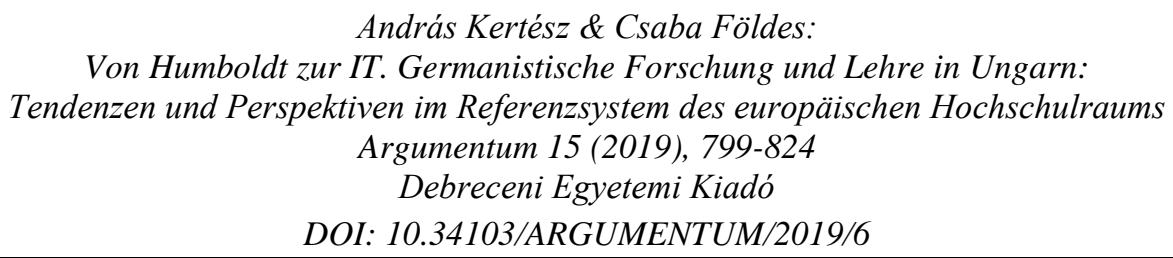

diese Sprache für ihre berufliche Laufbahn über ein nennenswertes Potenzial verfügt (Tabelle $7 b)$.

Wie in Abschnitt 5.3.3 angesprochen, haben 54\% der Befragten ein Austauschstudium im deutschsprachigen Raum vor. Dass unter den Gründen gegen ein mögliches Austauschstudium die Verlängerung des Studiums an erster Stelle genannt wird, scheint ebenfalls durch die lokalen Umstände der Befragten beeinflusst worden zu sein. Obwohl finanzielle Gründe mit einem relativ hohen Prozentsatz explizit genannt werden, hat auch die eventuelle Verlängerung des Studiums zweifellos für viele Studierende unerwünschte finanzielle Konsequenzen.

\subsubsection{Einschätzung der Effektivität des Germanistikstudiums seitens der Lehrenden und Bewertung der Germanistikausbildung seitens der Studierenden}

Die Antworten auf die Frage, wie lange die Studierenden schon Deutsch gelernt haben, wurden 2008 nicht ausgewertet, aber die Umfrage 2019 lässt relevant erscheinende Schlüsse zu. Obwohl Tabelle 5 gezeigt hat, dass fast zwei Drittel der Studierenden (63\%) mindestens seit 10 Jahren Deutsch lernen, sind die Erfahrungen der Dozenten des Instituts für Germanistik im Hinblick auf die Sprachkenntnisse der Studierenden eher negativ: Selbst Studierende der höheren Semester, die laut Umfrage seit etwa drei bis fünf Jahren an der Universität Germanistik studieren und davor weitere fünf Jahre oder noch länger Deutsch gelernt haben, verfügen über eine mangelhafte Sprachkompetenz. Es kann dafür mehrere Gründe geben, die allerdings aus der Umfrage selbst nicht hervorgehen. Welche Begründung auch immer zutreffen mag, Tabelle 5 legt nahe, dass die Diskrepanz zwischen der Dauer der Beschäftigung mit dem Deutschen und dem sprachlichen Niveau der Absolventen gründlich analysiert und soweit wie möglich durch komplexe sprachpolitische und interuniversitäre Maßnahmen behoben werden sollte.

In den Daten der Tabelle 8 über die Anwendung der Deutschkenntnisse außerhalb des Studiums spielen offensichtlich die Medien eine herausragende Rolle (siehe Zeilen 1, 2 und 11). Persönliche Kontakte zu Muttersprachlern sind zwar vorhanden, aber sie kommen weniger häufig vor (Zeilen 3 und 7). Dieser Befund scheint der oben genannten Annahme, dass 2019 mehr Studierende die Möglichkeit hatten, Erfahrungen mit deutschsprachigen Ländern zu sammeln als 2008, zu widersprechen; dieser Widerspruch lässt sich aufgrund der uns zur Verfügung stehenden Daten nicht auflösen.

\subsubsection{Einstellung zur Mehrsprachigkeit und zur europäischen Identität}

Die Daten in den Tabellen 9a, 9b, 10a, 10b, 11a sowie 11b zeigen eine eindeutige Tendenz: Unter den drei verschiedenen Aspekten wird Mehrsprachigkeit zunehmend positiv bewertet. Allerdings scheint die Beurteilung der Rolle des Englischen in den Tabellen 12a,12b, 13a und $13 \mathrm{~b}$ auf den ersten Blick mit der Rolle, die dem Deutschen im Studium und im Beruf zugeschrieben wurde, nicht zu harmonieren. Während nämlich die Daten in den Tabellen 6a, 6b, 7a und 7b auf die zunehmende Relevanz des Deutschen in diesen Domänen hindeuten, weisen hingegen die Daten in den Tabellen 12a, 12b, 13a und 13b auf beiden Gebieten dem Englischen einen steigenden Stellenwert zu. Dieser Unterschied verkörpert aber keinen Widerspruch, weil sich die Fragen, deren Antworten in den Tabellen 6-7 angegeben worden sind, auf lokale Bedingungen beziehen, während diejenigen, deren Beantwortung in den 


\author{
András Kertész \& Csaba Földes: \\ Von Humboldt zur IT. Germanistische Forschung und Lehre in Ungarn: \\ Tendenzen und Perspektiven im Referenzsystem des europäischen Hochschulraums \\ Argumentum 15 (2019), 799-824 \\ Debreceni Egyetemi Kiadó \\ DOI: 10.34103/ARGUMENTUM/2019/6
}

Tabellen 12a, 12b, 13a und 13b enthalten sind, das Referenzsystem des europäischen Hochschulraums ansprechen.

Vergleicht man die Werte in den Tabellen 9a, 9b, 10a, 10b, 11a und 11b mit denen in den Tabellen 14a und 14b, so ergibt sich, dass unter den Befragten auf der einen Seite die positive Beurteilung der Mehrsprachigkeit im Hinblick auf die Förderung der europäischen Identität im letzten Jahrzehnt deutlich gestiegen ist, aber auf der anderen Seite auch der in den Tabellen 15a und 15b erwähnte Nachteil 2019 stärker wahrgenommen wurde als 2008 (2008: $29 \%$ und 2019: $37 \%$ ). Dieses Bild wird allerdings durch die Daten der Tabelle 18, nach denen insgesamt $87 \%$ der Befragten es als wahrscheinlich oder sehr wahrscheinlich erachten, dass die Bedeutung des Deutschen im Berufsleben steigen werde, weiter differenziert.

\title{
6 Schlussfolgerungen
}

Nachdem die allgemeine Tendenz im Bereich der Neukonzipierung von Forschung und Lehre in Ungarn innerhalb des europäischen Hochschulraums skizziert und diese mit Blick auf die gegenwärtige ungarische Wissenschaftspolitik in Bezug auf die Geisteswissenschaften spezifiziert sowie eine Meinungsumfrage unter Germanistikstudenten der Universität Debrecen durchgeführt und ausgewertet wurde, können wir auf die Beantwortung der eingangs gestellten beiden Fragen eingehen.

Was die erste Frage betrifft, haben Abschnitte 2 bis 4 einige relevante Trends der europäischen und der ungarischen Hochschulpolitik zusammengefasst und ihre Konsequenzen für die ungarische Germanistik angedeutet. Wie zu erkennen war, fokussieren diese Tendenzen auf die Ablösung des Humboldtschen Hochschulsystems durch das Vorhaben, Forschung und Lehre wirtschaftsnah zu gestalten und den Schlüsselbegriff der Innovation in den Vordergrund zu stellen. Die ungarische Germanistik scheint sich gegenwärtig an diese Tendenzen nicht effektiv genug angepasst zu haben, was zu einer krisenartigen Situation im Germanistikstudium führt. Zum einen scheinen manche traditionellen Werte der Germanistik - d.h. die Ausbildung der Studierenden auf den Gebieten der Sprachwissenschaft, der Literaturgeschichte und des kulturellen Erbes - mit den erwähnten generellen hochschulpolitischen Tendenzen nicht im Einklang zu stehen. Auf der anderen Seite hat die ungarische germanistische Ausbildung zwar wichtige Schritte getan, um den neuen Anforderungen nachzukommen, diese haben bislang jedoch nicht zu der erwünschten Effektivität geführt.

Setzt man diese generelle Konsequenz mit der Auswertung der Meinungsumfrage in Beziehung, so ergibt sich ein Befund, der den gegenwärtigen Zustand der ungarischen Germanistik weiter differenziert. Die Umfrage legt nämlich folgende Schlüsse nahe:

(a) Die Art und Weise, wie die Studierenden die Bedeutung ihres Fachs für ihr Studium und für ihren Beruf beurteilen, widerspiegelt unmittelbar die lokalen Gegebenheiten in der Stadt Debrecen bzw. an der Universität Debrecen. Gegenwärtig gilt letztere als eine der wenigen Vorzeigeuniversitäten, die auf dem Wege sind, sich zu einer unternehmerischen Universität zu entwickeln. Sie erhielt eine enorme finanzielle Unterstützung von der Regierung, die die Innovation und somit die wirtschaftliche Tätigkeit der Universität fördern soll. Während Lehrende in zahlreichen eher kleineren Unternehmen, vor allem im Bereich der Medizin und der Ingenieurwissenschaften beteiligt sind, gilt das Interesse von Studierenden eher den groBen internationalen Unternehmen (wie etwa BMW oder Telekom), bei denen sie eine gut be- 


\author{
András Kertész \& Csaba Földes: \\ Von Humboldt zur IT. Germanistische Forschung und Lehre in Ungarn: \\ Tendenzen und Perspektiven im Referenzsystem des europäischen Hochschulraums \\ Argumentum 15 (2019), 799-824 \\ Debreceni Egyetemi Kiadó \\ DOI: 10.34103/ARGUMENTUM/2019/6
}

zahlte Stelle anstreben. Die bereits erörterte Tendenz, dass Germanistikstudierende zunehmend als Manager in der Wirtschaft - statt wie früher als Lehrer oder Übersetzer - tätig sein möchten, zeigt, dass die Studierenden ihre Zukunftspläne relativ schnell an die Leitwerte einer im Entstehen begriffenen unternehmerischen Universität angepasst haben.

(b) Die Sichtweise der Studierenden, die in erster Linie durch nutzenorientierte und kommerzielle Werte und weniger durch reines Interesse an der deutschen Sprache und Kultur geprägt ist, scheint mit der aktuellen Hochschulpolitik ebenfalls übereinzustimmen.

(c) Die Veränderung im Wertesystem der Studierenden widerspricht allerdings dem Niveau ihrer Sprachkenntnisse. Wenn ihre Ausbildung nicht mehr auf Sprach-, Literatur- und Kulturwissenschaft fokussiert, sondern auf die Vorbereitung ihrer Tätigkeit in der Wirtschaft, dann sollte die Germanistenausbildung der Förderung ihrer praktischen Sprachkenntnisse den Vorrang geben. Dem ist es aber nicht so, wie bereits im Abschnitt 5.4 angemerkt wurde.

(d) Wichtige Schlussfolgerungen ergeben sich auch, wenn man die generellen Tendenzen der Hochschulpolitik mit den Ansichten der Studierenden über die Mehrsprachigkeit und über die europäische Identität in Beziehung setzt. Studierende halten Mehrsprachigkeit, wie gesagt, für einen wichtigen europäischen Wert und begrüßen eine europäische Identität, selbst wenn sie auch manche Nachteile der Mehrsprachigkeit artikulieren.

Diese Schlussfolgerungen legen nahe: Während die Urteile der Studierenden den Veränderungen in der Hochschulpolitik folgen, hinkt das akademische Curriculum der Germanisten hinter diesen Entwicklungen her. Was könnte man nun, um auf unsere zweite Frage in Abschnitt 1 zurückzukommen, in einer solchen widersprüchlichen Situation tun? Unseren Beitrag wollen wir mit der Auflistung einiger Antwortmöglichkeiten schließen.

(a) Wenn Germanistik seitens der Regierung nicht mehr den traditionellen akademischen Ansprüchen gemäß gefördert wird, kommt man nicht umhin, das Konzept der Lehre der gegenwärtigen Forschungspolitik anzupassen. Wie groß dabei der Kompromiss sein sollte und sein darf und inwieweit die klassischen intellektuellen Werte mit kommerziellen Zielsetzungen noch zu vereinbaren sind - dies sind natürlich zentrale Fragen, die ein tiefgreifendes Nachdenken erfordern.

(b) Die oben erhobene Forderung nach Anpassung reicht mit Sicherheit nicht. Was man darüber hinaus nicht aufgeben sollte, ist der Versuch, wissenschaftspolitische Präferenzen in Ungarn zu beeinflussen. Die ungarische Germanistik sollte Instrumente finden, ihre Interessen so zu vertreten, dass sie die Entscheidungsträger erreicht. Wenn Germanistik-Absolventen nicht mehr als Lehrer in Grundschulen, Gymnasien und Sprachschulen, sondern als Manager in der freien Wirtschaft tätig sein wollen oder eben deutschsprachige IT-Kommunikation betreiben möchten, wird es wohl demnächst an Deutschlehrern fehlen, wodurch der schulische Deutschunterricht sowohl qualitativ als auch quantitativ Schaden nimmt.

(c) Hierzu ist eine verstärkte Kooperation der Gesellschaft ungarischer Germanisten $(\mathrm{GuG})$ mit anderen geisteswissenschaftlichen Verbänden, eventuell sogar die Gründung eines gesamt-geisteswissenschaftlichen Dachverbandes notwendig, den die Vertreter der Regierung als Verhandlungspartner anerkennen. Solange sich die Aufsplitterung geisteswissenschaftlicher Forschung auch in der Aufsplitterung der Verbände niederschlägt, besteht kaum eine Chance für eine effektive Interessenvertretung.

(d) Es sei auch erwähnt, dass die oft wahrnehmbare Einordnung der Geisteswissenschaften als „nutzlos“ mit rationaler Argumentation leicht widerlegt werden kann - und diese Argu- 


\author{
András Kertész \& Csaba Földes: \\ Von Humboldt zur IT. Germanistische Forschung und Lehre in Ungarn: \\ Tendenzen und Perspektiven im Referenzsystem des europäischen Hochschulraums \\ Argumentum 15 (2019), 799-824 \\ Debreceni Egyetemi Kiadó \\ DOI: 10.34103/ARGUMENTUM/2019/6
}

mentation sollte die Entscheidungsträger mithilfe entsprechender Werbe- und Lobbytätigkeit erreichen. ${ }^{6}$

(e) Die oben erörterten Maßnahmen der gegenwärtigen ungarischen Hochschulpolitik stellen eine extreme Verwirklichung von Präferenzen des europäischen Hochschulraums dar; extrem deshalb, weil sie der Differenziertheit des Letzteren nicht subtil folgt, sondern lediglich seine radikalsten Richtlinien mechanisch übernimmt (vgl. Pléh 2006, Polónyi 2005 und Pritz et al. 2005). Der europäische Hochschulraum ist nämlich wesentlich feiner gefächert als diese vereinfachte Sichtweise. Beispielsweise muss man, selbst wenn das Stichwort „Innovation" als die wichtigste Zielsetzung der Hochschulpolitik angesehen wird, anerkennen, dass deren Ergebnisse nur in einem komplexen gesellschaftlichen Umfeld angemessen Anwendung finden können. Es ist mithin leicht einzusehen, dass der Germanistik in dieser Hinsicht eine Schlüsselrolle zukommen könnte, weil sie zur Erfassung, zur Beschreibung sowie auch - und besonders - zum Begreifen essenzieller kultureller Zusammenhänge und interkultureller Bewegungen und Phänomene in einem mehrfach kodierten europäischen Raum substanzielle Dienste leisten kann, ohne die auch die vielbeschworene Innovation nicht möglich ist. Die ungarische Germanistik könnte und sollte in diesem Prozess selbst auf gesamteuropäischer Ebene eine proaktive Rolle spielen.

\title{
Literatur
}

Enyedi, György (2001): A társadalomtudományok százada [Das Jahrhundert der Gesellschaftswissenschaften]. Magyar Tudomány 162.2, 170-175.

Enyedi, György (2004): A bölcsészeti és a társadalomtudományok a Magyar Tudományos Akadémián [Geisteswissenschaften und Sozialwissenschaften an der Ungarischen Akademie der Wissenschaften]. Magyar Tudo mány 165.12, 1382-1386.

Földes, Csaba (2016): Position und Entwicklungsdynamik des Deutschen als Fremdsprache international - im Blick der angewandten Linguistik. Glottodidactica. An International Journal of Applied Linguistics 43.2, 13-38. https://doi.org/10.14746/gl.2016.43.2.01.

Goltschnigg, Dietmar (Hrsg.) (2017): Wege des Deutschen. Deutsche Sprache und Germanistik-Studium aus internationaler Sicht. Tübingen: Stauffenburg.

Höffe, Otfried (2005): Vom Nutzen des Nutzlosen. Zur Bedeutung der Philosophie im Zeitalter der Ökonomisierung. Deutsche Zeitschrift für Philosophie 53.5, 667-678. https://doi.org/10.1524/dzph.2005.53.5.667.

Kertész, András (2008): Sind germanistische Forschungen noch zu retten? Bemerkungen zur Situation der Geisteswissenschaften im Ungarn der Jahrtausendwende. In: Tichy, Ellen \& Masát, András (Hrsg.): Jahrbuch der ungarischen Germanistik 2007. Bonn: DAAD/Budapest: GUG, 30-44.

Kuhn, Thomas S. (1970): The Structure of Scientific Revolutions. Chicago: Chicago University Press.

6 Ein lehrreiches und anschauliches Beispiel für diese Art der Argumentation ist der Aufsatz von Höffe (2005). Analoge Argumente ließen sich zur Verteidigung des Nutzens germanistischer Forschungen leicht konstruieren. Dass die Argumentation mit entsprechendem Engagement der Betroffenen u. U. spektakuläre Ergebnisse erzielen kann, bezeugt etwa das in Rózsa (2008) geschilderte Beispiel. 
Masát, András \& Lőkös, Péter (2017): Germanistik in Ungarn. Klassische Bildung und/oder Arbeitsmarktorientierung. Mittelungen des Deutschen Germanistenverbandes 64.1, 57-62. https://doi.org/10.14220/mdge.2017.64.1.57.

Mezei, Zsuzsa (2009): Zur Rolle des Deutschen in der europäischen Identität. Ergebnisse einer Umfrage unter Germanistikstudenten der Universität Debrecen, Ungarn. In: Argumentum (Debrecen) 5, 44-72.

Pléh, Csaba (2006): A tudomány szabadsága a pénz világában [Die Freiheit der Wissenschaft in der Welt des Geldes]. Magyar Tudomány 167.7, 879-885.

Polónyi, István (2005): Zsákban táncolva, avagy az egyetemi innovációs stratégia sajátosságai [Im Sack tanzend, oder: die Eigenheiten der Innovationsstrategie an Universitäten]. Competitio 4.1, 201-212. https://doi.org/10.21845/comp/2005/1/12.

Pritz, Pál et al. (szerk.) (2005): Társadalomtudomány - Tudománypolitika az MTA-ról nézve. A 2005. szeptember 30-i konferencia háttéranyaga [Gesellschaftswissenschaften - Wissenschaftspolitik aus der Sicht der Ungarischen Akademie der Wissenschaften. Hintergrundmaterial der Tagung vom 30.09.2005]. Budapest: Magyar Tudományos Akadémia.

Rózsa, Erzsébet (2008): A Berlin-Brandenburgi Tudományos Akadémia interdiszciplináris humánprojektje [Das interdisziplinäre Humanprojekt der Berlin-Brandenburgischen Akademie der Wissenschaften]. Magyar Tudomány 169.3, 343-351.

\author{
András Kertész \\ Universität Debrecen, Institut für Germanistik \\ und MTA-DE-SZTE Forschungsstelle für Theoretische Linguistik \\ Egyetem tér 1. \\ H-4032 Debrecen \\ Ungarn \\ kertesz.andras@arts.unideb.hu \\ Csaba Földes \\ Universität Erfurt \\ Philosophische Fakultät \\ Lehrstuhl für Germanistische Sprachwissenschaft \\ Nordhäuser Str. 63 \\ D-99089 Erfurt \\ Deutschland \\ foeldes@foeldes.eu
}

